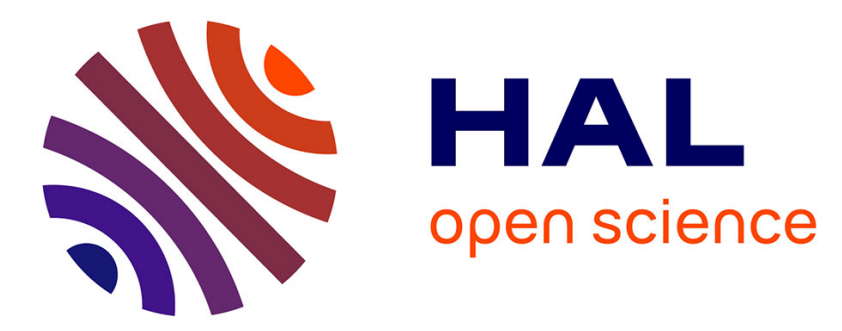

\title{
Lagrangian Mixing Models for Turbulent Combustion: Review and Prospects
}

Cesar Celis, Luís Fernando Figueira da Silva

\section{To cite this version:}

Cesar Celis, Luís Fernando Figueira da Silva. Lagrangian Mixing Models for Turbulent Combustion: Review and Prospects. Flow, Turbulence and Combustion, 2015, 94 (3), pp.643-689. 10.1007/s10494015-9597-1 . hal-03313655

\section{HAL Id: hal-03313655 \\ https://hal.science/hal-03313655}

Submitted on 20 Aug 2021

HAL is a multi-disciplinary open access archive for the deposit and dissemination of scientific research documents, whether they are published or not. The documents may come from teaching and research institutions in France or abroad, or from public or private research centers.
L'archive ouverte pluridisciplinaire HAL, est destinée au dépôt et à la diffusion de documents scientifiques de niveau recherche, publiés ou non, émanant des établissements d'enseignement et de recherche français ou étrangers, des laboratoires publics ou privés. 


\title{
Lagrangian Mixing Models for Turbulent Combustion: Review and Prospects
}

\author{
Cesar Celis ${ }^{1}$, Luís Fernando Figueira da Silva \\ Department of Mechanical Engineering, Pontifícia Universidade Católica do Rio de Janeiro \\ Rua Marquês de São Vicente 225, Rio de Janeiro, RJ 22453-900, Brazil
}




\section{Abstract}

Compared to other simulation approaches utilized for modeling turbulent reacting flows with detailed chemistry, probability density function (PDF) methods offer several advantages. This is because the changes in fluid composition due to convection and reaction processes can be treated exactly. PDF methods require however closure models for the mixing process representing the transport of the PDF owing to molecular diffusion. Over the years several mixing models with different degrees of complexity have been developed. A review of the main Lagrangian mixing models for turbulent combustion developed so far is presented in this work. This review includes models where the composition of a particle changes (i) essentially independently of the composition associated with the other particles, and (ii) through direct interaction with other particles. The main advantages and shortcomings of the mixing models reviewed are highlighted accordingly. Because Lagrangian Monte Carlo techniques are usually used for solving PDF transport equations, a particular emphasis is put on their corresponding particle implementation. The mixing models review is preceded by a section highlighting the mathematical formulation associated with the use of PDF methods for turbulent reacting flows. In the last part of the article both comparative results of mixing models performance and prospects for the mixing models are discussed. Despite the effort that has been devoted to the development of more capable mixing models, currently there is no mixing model presenting all desirable characteristics. Even more, there are significant differences in the results obtained when different mixing models are utilized, especially when higher order scalar statistics are accounted for. Therefore work still needs to be carried out in order to develop a mixing model satisfying all desirable characteristics expected from these models. Several avenues can be further explored in order to achieve this goal. These potential routes include those accounting for spatial scalar structures and both scalar length and turbulent frequency scales distributions. Other approaches based on competitive mixing, manifold-based features and Lagrangian coherent structures have also the potential to further improve upon existing mixing models. The development of a sound mixing model will allow eventually removing one of the largest sources of modeling uncertainty in PDF-based computations. 
Keywords: Lagrangian mixing models, Probability density function methods, Turbulent combustion. 


\section{Contents}

1 Introduction 9

2 Mathematical Formulation 11

2.1 Governing Equations 11

2.2 PDF Transport Equations 13

$\begin{array}{lll}2.3 & \text { Lagrangian Particle Equations } & 16\end{array}$

3 State-of-the-art of Mixing Models 19

3.1.1 Interaction by Exchange with the Mean (IEM) 22

3.1.2 Coalescence and Dispersion (CD) 24

3.1.3 Mapping Closure (MC) 26

3.1.4 Euclidean Minimum Spanning Tree (EMST) 27

3.1.5 Multiple Mapping Conditioning (MMC) 29

3.1.6 Shadow-Position Mixing Model (SPMM) 31

3.1.7 Parameterized Scalar Profile (PSP) 32

3.1.8 Other Mixing Models 34

4 Mixing Models Comparative Results 35

$5 \quad$ Prospects for Mixing Models $\quad 51$

6 Conclusions 56

7 Acknowledgments $\quad 57$

8 References $\quad 58$ 


\section{Nomenclature}

Physical constants

$\mathrm{R}_{0} \quad$ Universal gas constant

Variables

$a_{m} \quad$ Model parameter

A Stochastic particle acceleration (model)

$b_{m} \quad$ Model parameter

B Rate of attraction of stochastic particles

$B_{v} \quad v^{\text {th }}$ spanning tree edge coefficient

c Reaction progress variable

$C_{\phi}, C_{\phi}^{\prime} \quad$ Model parameters

$C_{0}, C_{\omega} \quad$ Model parameters

$\hat{d} \quad$ Normalized distance

$\tilde{f} \quad$ Reference space location

$\langle f(x, t)\rangle_{1} \quad$ Filtered value of $f(x, t)$

$\langle f(x, t)\rangle_{L} \quad$ Favre filtered value of $f(x, t)$

$\mathrm{F}\left(\mathrm{x}^{\prime}, \mathrm{x}\right) \quad$ Filter function

$\mathcal{F}_{\phi} \quad$ Joint composition probability density function

$\mathcal{F}_{u \phi} \quad$ Joint velocity-composition probability density function

$\mathcal{F}_{\omega u \phi} \quad$ Joint frequency-velocity-composition probability density function

$\mathrm{g}(\eta) \quad$ Standard Gaussian density function

h Random parameter

$\mathrm{J}_{\mathrm{i}}^{\alpha} \quad$ Scalar flux of species $\alpha$

$\mathrm{k} \quad$ Subgrid scale kinetic energy

$\mathrm{L}_{\mathrm{f}} \quad$ Reference (space) characteristic scale

$\mathrm{L}_{\mathrm{x}} \quad$ Physical (space) characteristic scale

$m_{v}, n_{v} \quad$ Stochastic particles corresponding to $v^{\text {th }}$ spanning tree edge

$\mathrm{M}_{\mathrm{ij}} \quad$ Stochastic particles interaction matrix

$\mathrm{N}_{\mathrm{p}} \quad$ Total number of stochastic particles

$N_{p}^{\prime} \quad$ Number of stochastic particles

$\mathrm{N}_{\mathrm{s}} \quad$ Chemical species total number 


$\begin{array}{ll}\mathrm{p} & \text { Dynamic pressure } \\ \mathrm{P} & \text { Pressure } \\ \mathrm{P}_{0} & \text { Thermodynamic pressure } \\ \mathrm{R} & \text { Shadow particle displacement } \\ \mathrm{S}_{\alpha} & \text { Chemical source term of species } \alpha \\ \mathrm{t} & \text { Time } \\ \mathrm{t}, \mathrm{t}_{+} & \text {Time stamp parameters } \\ \mathrm{T} & \text { Temperature } \\ \mathrm{T}_{\mathrm{L}} & \text { Lagrangian integral time scale } \\ \mathrm{u} & \text { Velocity vector } \\ \mathrm{u}_{\mathrm{i}} & \text { Velocity vector component } \\ \mathrm{W} & \text { Stochastic particle weight } \\ \mathrm{W} & \text { Wiener-Lévy process } \\ \mathrm{W}_{\alpha} & \text { Molecular weight of species } \alpha \\ \mathrm{x} & \text { Position vector } \\ \mathrm{x}_{\mathrm{i}} & \text { Position vector component } \\ \mathrm{X} & \text { Logarithm of turbulence frequency } \\ \mathrm{Y} & \text { Residence to chemical time scale ratio } \\ \mathrm{Y}_{\alpha} & \text { Mass fraction of species } \alpha \\ \mathrm{Z} & \text { Shadow particle position } \\ & \text { Residence to turbulence time scale ratio } \\ & \end{array}$

Greek-letter variables

$\begin{array}{ll}\alpha_{\mathrm{p}} & \text { Model parameter or mixing extent } \\ \Gamma & \text { Molecular diffusion coefficient } \\ \Gamma_{\mathrm{t}} & \text { Turbulent diffusion coefficient } \\ \delta & \text { Dirac delta function } \\ \delta_{\mathrm{ij}} & \text { Kronecker delta } \\ \zeta & \text { Fine-grained density } \\ \eta & \text { Sample space coordinate (Gaussian density function) } \\ \theta & \text { Turbulence frequency sample space } \\ \Theta & \text { Turbulence frequency model (stochastic particles) } \\ \lambda & \text { Relative localization parameter or one dimensional profile length scale } \\ \mu & \text { Fluid dynamic viscosity } \\ \xi & \text { Mixture fraction } \\ \xi^{\prime}, \xi^{\prime \prime} & \text { Mixture fraction root mean square }\end{array}$




$\begin{array}{ll}\rho & \text { Density } \\ \sigma & \text { Root mean square velocity } \\ & \text { Standard deviation } \\ \varsigma & \text { Local anisotropy parameter } \\ \tau & \text { Turbulence time scale } \\ \tau_{\mathrm{ij}} & \text { Viscous stress tensor } \\ \tau_{r e s} & \text { Residence time } \\ \tau_{m i x} & \text { Mixing time } \\ \tau_{\phi} & \text { Mixing time scale } \\ v & \text { Velocity sample space } \\ \phi & \text { Composition vector } \\ \hat{\phi} & \text { Weighted two-particle average scalar value } \\ \Phi & \text { Mixing model (stochastic particles) } \\ \psi & \text { Composition sample space } \\ \omega & \text { Turbulence frequency } \\ \widehat{\omega} & \text { Local turbulence frequency } \\ \Omega & \text { Subgrid scale mixing frequency }\end{array}$

Subscripts

c Weighted average

$\alpha \quad$ Chemical species or composition vector component

- $\quad$ One dimensional profile minimum value

$+\quad$ One dimensional profile maximum value

Superscripts

t Time

(p), (q) Stochastic particle p or q

$\alpha \quad$ Chemical species or composition vector component

* Any stochastic particle

(i) $\mathrm{i}^{\text {th }}$ stochastic particle

Abbreviations

BI Burning indexes

CD Coalescence and dispersion (mixing model) 


$\begin{array}{ll}\text { CDF } & \text { Cumulative density function } \\ \text { CFD } & \text { Computational fluid dynamics } \\ \text { CMC } & \text { Conditional moment closure } \\ \text { DNS } & \text { Direct numerical simulation } \\ \text { EIEM } & \text { Extended IEM (mixing model) } \\ \text { ELM } & \text { Extended Langevin (mixing) model } \\ \text { EMST } & \text { Euclidean minimum spanning tree (mixing model) } \\ \text { FDF } & \text { Filtered density function } \\ \text { FMDF } & \text { Filtered mass density function } \\ \text { FP } & \text { Fokker-Planck } \\ \text { IECM } & \text { Interaction by exchange with the conditional mean (mixing model) } \\ \text { IEM } & \text { Interaction by exchange with the mean (mixing model) } \\ \text { ISAT } & \text { In situ adaptive tabulation } \\ \text { LCS } & \text { Lagrangian coherent structures } \\ \text { LES } & \text { Large eddy simulation } \\ \text { LM } & \text { Langevin (mixing) model } \\ \text { LMSE } & \text { Linear mean-square estimation (mixing model) } \\ \text { MC } & \text { Mapping closure (mixing model) } \\ \text { MCD } & \text { Modified CD (mixing model) } \\ \text { MMC } & \text { Multiple mapping conditioning } \\ \text { PaSR } & \text { Partially stirred reactor } \\ \text { PDF } & \text { Probability density function } \\ \text { PSP } & \text { Parameterized scalar profile (mixing model) } \\ \text { RANS } & \text { Reynolds-averaged Navier Stokes } \\ \text { RAS } & \text { Reynolds-averaged simulation } \\ \text { RHS } & \text { Right hand side } \\ \text { RIEM } & \text { Refined IEM (mixing model) } \\ \text { RMS (rms) } & \text { Root mean square } \\ \text { SDE } & \text { Stochastic differential equation } \\ \text { SGS } & \text { Subgrid scale } \\ \text { SIEM } & \text { Stochastic IEM (mixing model) } \\ \text { SLM } & \text { Simplified Langevin model } \\ \text { Shadow-position mixing model } \\ \text { Velocity conditioned IEM (mixing model) } \\ \text { VPM-shadow-position mixing model } \\ \text { VMM }\end{array}$




\section{Introduction}

Global economy is expected to grow in the following years, which implies that the world demand for energy will augment as well. Indeed, compared to the amount corresponding to 2011, global energy demand is expected to increase near one-third by 2035 [1]. The so-called clean energy should be however the main driver due to the increasing public awareness about the anthropogenic contribution to global warming. Under clean energy denomination is understood here both energy efficiency, and clean energy supply options such as those based on renewable energy sources. The fact that combustion processes are responsible for more than $80 \%$ [1] of the energy used by humanity suggests that they are and will continue to be vital for world economy and crucial in our daily activities. Designing practical combustion systems that are both efficient and environmentally friendly requires, in general, an in-depth knowledge of the tight coupling between turbulent fluctuations, chemical reactions and mixing.

Probability density function (PDF) methods offer compelling advantages for modeling chemically reacting turbulent flows. They provide indeed an effective solution to the closure problems that arise from averaging/filtering, in the instantaneous governing equations, both nonlinear chemical source terms and terms corresponding to other one-point physical processes (e.g. radiative emission) [2]. For details about averaging/filtering processes and closure problems associated with transport equations governing turbulent reacting flows, the reader is referred to [3,4]. Despite the on-going progress in computational fluid dynamics (CFD) modeling for inert-scalar transport, the treatment of turbulent reacting flows offers unique challenges. While turbulent transport of a chemically inert scalar can often be successfully described by a small set of statistical moments, the same is not true for scalar fields strongly coupled through the chemical source term, which appears in the reacting scalar transport equations $[3,4]$. Even so, because the chemical source term depends only on the local molar concentrations and temperature, knowledge of the one-point, one-time composition PDF at all points in the flow could suffice to predict the mean chemical source term [5]. A particular formulation used for the modeling of turbulent reacting flows is that known as 'transported PDF' method. This approach involves solving a transport equation for the joint-scalar PDF in which the chemical source term appears in closed form. Specifically, 
one models and solves a transport equation that governs the evolution of the one-point, one-time PDF for a set of variables that determines the local thermochemical and/or hydrodynamic state of the reacting system [2].

The PDF transport equation solved is in general high-dimensional requiring thus the use of Monte Carlo techniques. Both Lagrangian and Eulerian Monte Carlo approaches have been utilized for solving this PDF transport equation. Lagrangian methods [5], the current mainstream approach, rely on the use of notional particles that evolve according to stochastic ordinary differential equations. In Eulerian Monte Carlo approaches [6-8], the notional particles are replaced by notional Eulerian fields that evolve according to stochastic partial differential equations. In addition to these stochastic approaches, deterministic Eulerian field PDF methods [4], based on sophisticated assumed PDF shapes, have been used as well to solve modelled PDF transport equations $[9,10]$. The advantages and disadvantages of each of these PDF methods in terms of accuracy and computational requirements are discussed in $[9,10]$. Regarding specifically to stochastic particle-based Lagrangian schemes, the two main approaches commonly utilized, in the case of low Mach number reacting flows, involve composition PDF and velocity-composition PDF methods [4]. When the particular PDF method does not include information about the turbulent velocity fields (as in the case of the joint composition PDF), a separate flow model is required. Hybrid grid/particle approaches [11-13] are often used in these situations. In these schemes the (grid-based) Eulerian flow model - involving large eddy simulation (LES), for example $[14,15]-$ provides the velocity and turbulence fields, and the (particle-based) Lagrangian scheme evolves the reactive scalars. The coupling between the Eulerian and Lagrangian schemes is achieved by transferring the mean fields used to advance the respective equations. This type of hybrid approach has been successfully used for studying several experimental configurations [16-19].

In turbulent reactive flows, the fluid composition at a point changes with time due to convection, mixing (i.e., molecular transport) and reaction [20,21]. PDF methods have proved successful mainly because, in these modeling approaches, the last of these processes can be treated exactly. In addition in velocity PDF or velocity-scalar PDF methods convection is also in closed form. In PDF transport equations nevertheless, mixing requires a closure model. Mixing models, the main subject of this work, are used thus for the purposes of closing the PDF transport equations. Over the last 50 years or 
so several mixing models with different degrees of complexity have been developed. For brief reviews on some of the early mixing models the reader is referred to [22-25]. As it will be shown in Sections 3 and 4, despite the efforts made in this direction, there is no mixing model satisfying all requirements usually associated with these models. In this work a review of the main mixing models developed so far for turbulent reacting flows is presented. The main advantages and shortcomings of the mixing models reviewed are highlighted accordingly. Because Lagrangian Monte Carlo techniques are commonly used for solving PDF transport equations, a particular emphasis is put on their corresponding particle implementation. The mixing models review (Section 3) is preceded by a brief recall of the mathematical formulation associated with the use of Lagrangian PDF methods for turbulent reacting flows (Section 2). In the last part of the article both comparative results of mixing models performance (Section 4) and prospects for mixing models development (Section 5) are discussed.

\section{Mathematical Formulation}

In order to emphasize the context in which mixing models are utilized, the mathematical formulation associated with the use of PDF methods for the modeling of turbulent reacting flows is summarized in this section. The transport equations governing this type of flows are initially indicated. This is followed by a description of the transported PDF equations utilized when using this approach. Finally the Lagrangian particle equations, which govern the evolution of the stochastic particles yielding the same one-point, one-time Eulerian PDF as the real fluid particle system, are described.

\subsection{Governing Equations}

Low Mach number turbulent reacting flows with variable density are considered here for the sake of simplicity. These flows are governed by the following transport equations (summation convention applies over repeated indices within a term) [26,27],

$$
\frac{\partial \rho}{\partial t}+\frac{\partial \rho u_{j}}{\partial x_{j}}=0
$$




$$
\begin{gathered}
\frac{\partial \rho u_{i}}{\partial t}+\frac{\partial \rho u_{j} u_{i}}{\partial x_{j}}=-\frac{\partial p}{\partial x_{i}}+\frac{\partial \tau_{j i}}{\partial x_{j}}, \quad i=1,2,3, \\
\frac{\partial \rho \phi_{\alpha}}{\partial t}+\frac{\partial \rho u_{j} \phi_{\alpha}}{\partial x_{j}}=-\frac{\partial J_{j}^{\alpha}}{\partial x_{j}}+\rho S_{\alpha}, \quad \alpha=1,2, \ldots, N_{s}+1,
\end{gathered}
$$

which, along an equation of state (where $R_{0}$ is the universal gas constant, $T$ temperature and $W_{\alpha}$ the molecular weight of species $\alpha$ ),

$$
P_{0}=\rho R_{0} T \sum_{\alpha=1}^{N_{S}} \frac{Y_{\alpha}}{W_{\alpha}}
$$

form a closed set of equations when supplemented by the adequate boundary conditions. These transport equations describe the spatial $\left(x \equiv x_{i}, i=1,2,3\right)$ and temporal $(t)$ variations of the fluid density $\rho(x, t)$, velocity $u \equiv u_{i}(x, t)$, pressure $P(x, t)-$ expressed as the sum of the thermodynamic $P_{0}(t)$ and dynamic pressures $p(x, t)$, and composition vector $\phi \equiv \phi_{\alpha}(x, t)$. The composition vector is given by the mass fractions $Y_{\alpha}$ of the $N_{s}$ chemical species compounding the gas-phase reacting system and enthalpy $\left(\alpha=1,2, \ldots, N_{s}+1\right)$. For a Newtonian fluid, with Fick's law of diffusion and equal molecular diffusion coefficients $\Gamma$, the viscous stress tensor $\tau_{i j}$ and the scalar flux $J_{j}^{\alpha}$ are given by,

$$
\begin{gathered}
\tau_{i j}=\mu\left(\frac{\partial u_{i}}{\partial x_{j}}+\frac{\partial u_{j}}{\partial x_{i}}\right)-\frac{2}{3} \mu \frac{\partial u_{k}}{\partial x_{k}} \delta_{i j} \\
J_{j}^{\alpha}=-\rho \Gamma \frac{\partial \phi_{\alpha}}{\partial x_{j}}
\end{gathered}
$$

where $\mu$ is the fluid dynamic viscosity. Finally the source terms $S_{\alpha}$, which are functions of the composition vector $\phi$, are directly related to both the chemical production rates of the corresponding species, and the thermal radiation heat transfer. In these governing equations it is considered that Soret and Dufour effects are negligible, as well as external heat source terms and volume forces. In the following radiative heat transfer is not accounted for as well.

Computational power has continuously increased over the years so nowadays it is possible to model more accurately turbulent reacting flows than in the past. This has enabled the use of LES-based approaches over RAS (Reynolds-averaged simulation) 
based ones for describing increasingly complex reacting systems. Transport equations in LES are usually filtered using a spatial filtering operation of the form [3],

$$
\langle f(x, t)\rangle_{l}=\int_{-\infty}^{+\infty} f\left(x^{\prime}, t\right) F\left(x^{\prime}, x\right) d x^{\prime}
$$

where $\langle f(x, t)\rangle_{l}$ is the filtered value of $f(x, t)$ and $F\left(x^{\prime}, x\right)$ represents the filter function. Accordingly a Favre filtered value of $f(x, t)$ can be defined as $\langle f(x, t)\rangle_{L}=$ $\langle\rho f\rangle_{l} /\langle\rho\rangle_{l}$. The filtered form of the governing equations is similar to the unfiltered one, Eqs. (1)-(3), so they are not explicitly indicated here but may be found elsewhere $[2,3,28]$. The only differences are related to the appearance of subfilter terms, also known as subgrid scale (SGS) terms, which are associated with the stress tensor and the scalar fluxes. PDF methods - or their analogues filtered density function (FDF) ones as they are often referred to in LES contexts - account for these SGS effects using probabilistic approaches.

\subsection{PDF Transport Equations}

Common practice currently involves the use of PDF methods in LES contexts so the form of the PDF transport equations described in this section corresponds to these particular situations. Notice however that, in order to reduce the proliferation of nomenclature, FDF methods accounting for SGS effects in a probabilistic manner will continue to be referred to as PDF methods. More specifically, the PDF-based formulation described in this section relates to filtered mass density function (FMDF) schemes [29] - an extension of FDF approaches for variable density flows - involving joint frequency-velocity-scalar, velocity-scalar or scalar PDFs. Accordingly, when using PDF methods for modeling turbulent reacting flows, PDFs that can be transported include joint frequency-velocity-composition PDFs, velocity-composition PDFs and composition PDFs. Each of these transported PDFs involves different levels of complexity and requirements. The joint frequency-velocity-composition PDF, $\mathcal{F}_{\omega u \phi}$, which includes a complete SGS statistical information, is formally defined as $[3,28]$, 


$$
\begin{aligned}
& \mathcal{F}_{\omega u \phi}(\theta, v, \psi ; x, t) \\
& =\int_{-\infty}^{+\infty} \rho\left(x^{\prime}, t\right) \zeta\left[\theta, v, \psi ; \omega\left(x^{\prime}, t\right), u\left(x^{\prime}, t\right), \phi\left(x^{\prime}, t\right)\right] F\left(x^{\prime}-x\right) d x^{\prime},
\end{aligned}
$$

where

$$
\begin{aligned}
& \zeta[\theta, v, \psi ; \omega(x, t), u(x, t), \phi(x, t)] \\
& \equiv \delta[\theta-\omega(x, t)] \prod_{i=1}^{3} \delta\left[v_{i}-u_{i}(x, t)\right] \prod_{\alpha=1}^{N_{S}+1} \delta\left[\psi_{\alpha}-\phi_{\alpha}(x, t)\right]
\end{aligned}
$$

is the fine-grained PDF [30,31] and $\delta$ the Dirac delta function. In these equations $\theta, v$ and $\psi$ represent, respectively, the sample spaces variables corresponding to turbulence frequency $(\omega)$, velocity and composition. The filtered value of any function of frequency, velocity, and/or scalars, $\hat{Q}$, is then obtained by integration over the corresponding sample spaces as follows,

$$
\begin{aligned}
& \langle\rho(x, t)\rangle_{l}\langle Q(x, t)\rangle_{L} \\
& =\iint \ldots \int_{-\infty}^{+\infty} \hat{Q}(\theta, v, \psi) \mathcal{F}_{\omega u \phi}(\theta, v, \psi ; x, t) d \theta d v d \psi .
\end{aligned}
$$

The exact transport equation for $\mathcal{F}_{\omega u \phi}$ is obtained by both (i) replacing in the temporal derivative of the fine-grained PDF,

$$
\frac{\partial \zeta}{\partial t}=-\left(\frac{\partial \zeta}{\partial \theta} \frac{\partial \omega}{\partial t}+\frac{\partial \zeta}{\partial v_{i}} \frac{\partial u_{i}}{\partial t}+\frac{\partial \zeta}{\partial \psi_{\alpha}} \frac{\partial \phi_{\alpha}}{\partial t}\right)
$$

the time derivatives of velocity and scalars, Eqs. (1)-(3), and (ii) performing an Eq. (8)based filtering operation. The final expression takes the form [28],

$$
\begin{aligned}
& \frac{\partial \mathcal{F}_{\omega u \phi}}{\partial t}+\frac{\partial v_{j} \mathcal{F}_{\omega u \phi}}{\partial x_{j}} \\
& =-\frac{\partial}{\partial \psi_{\alpha}}\left[S_{\alpha}(\psi) \mathcal{F}_{\omega u \phi}\right]+\frac{\partial}{\partial \psi_{\alpha}}\left[\left|\frac{1}{\rho(\phi)} \frac{\partial J_{j}^{\alpha}}{\partial x_{j}}\right| \theta, v,\left.\psi\right|_{l} \mathcal{F}_{\omega u \phi}\right] \\
& +\frac{\partial}{\partial v_{i}}\left[\left|\frac{1}{\rho(\phi)}\left(\frac{\partial p}{\partial x_{i}}-\frac{\partial \tau_{j i}}{\partial x_{j}}\right)\right| \theta, v,\left.\psi\right|_{l} \mathcal{F}_{\omega u \phi}\right]
\end{aligned}
$$




$$
-\frac{\partial}{\partial \theta}\left[\left\langle\frac{D \omega}{D t} \mid \theta, v, \psi\right\rangle_{l} \mathcal{F}_{\omega u \phi}\right]
$$

where $D / D t$ represents a material derivative. In Eq. (12) the conditional filter terms, $\langle\ldots \mid \theta, v, \psi\rangle_{l}$, are unclosed [28], which implies that models are required for modeling them. These open terms represent, specifically, from left to right, the PDF transport in (i) composition space due to molecular processes, (ii) velocity space due to pressure gradients and molecular process, and (iii) turbulence frequency space. By contrast the first RHS (right hand side) term of Eq. (12), which is associated with the chemical source term, appears in closed form. This implies that even complex chemical kinetics schemes can be treated by PDF methods without major difficulties. Hence their usefulness for modeling turbulent reacting flows.

A corresponding exact transport equation for the joint velocity-composition PDF, $\mathcal{F}_{u \phi}$,

$$
\mathcal{F}_{u \phi}(v, \psi ; x, t)=\int_{-\infty}^{+\infty} \mathcal{F}_{\omega u \phi}(\theta, v, \psi ; x, t) d \theta
$$

is obtained by integrating Eq. (12) over the frequency sample space [32],

$$
\begin{aligned}
& \frac{\partial \mathcal{F}_{u \phi}}{\partial t}+\frac{\partial v_{j} \mathcal{F}_{u \phi}}{\partial x_{j}} \\
& =-\frac{\partial}{\partial \psi_{\alpha}}\left[S_{\alpha}(\psi) \mathcal{F}_{u \phi}\right]+\frac{\partial}{\partial \psi_{\alpha}}\left[\left|\frac{1}{\rho(\phi)} \frac{\partial J_{j}^{\alpha}}{\partial x_{j}}\right| v,\left.\psi\right|_{l} \mathcal{F}_{u \phi}\right] \\
& +\frac{\partial}{\partial v_{i}}\left[\left\langle\frac{1}{\rho(\phi)}\left(\frac{\partial p}{\partial x_{i}}-\frac{\partial \tau_{j i}}{\partial x_{j}}\right)|v, \psi|_{l} \mathcal{F}_{u \phi}\right] .\right.
\end{aligned}
$$

Similarly, after Eq. (14) partial integration in velocity space, the transport equation for the joint composition PDF, $\mathcal{F}_{\phi}$, is expressed as [33]

$$
\begin{aligned}
& \frac{\partial \mathcal{F}_{\phi}}{\partial t}+\frac{\partial}{\partial x_{j}}\left[\left\langle u_{j} \mid \psi\right\rangle_{l} \mathcal{F}_{\phi}\right] \\
& =-\frac{\partial}{\partial \psi_{\alpha}}\left[S_{\alpha}(\psi) \mathcal{F}_{\phi}\right]+\frac{\partial}{\partial \psi_{\alpha}}\left[\left\langle\frac{1}{\rho(\phi)} \frac{\partial J_{j}^{\alpha}}{\partial x_{j}}|\psi|_{l} \mathcal{F}_{\phi}\right] .\right.
\end{aligned}
$$

As highlighted by Eq. (6), the second RHS terms of Eq. (12), (14) and (15), dealing with the joint PDF transport in composition space, require the knowledge of the 
transported scalar gradients conditional averages. The PDF transport equations described here are however formulated for one-point, one-time statistics so as to contain no gradient information. Mixing models are thus required for closing these specific open PDF (transport equation) terms. Additional information regarding the methodologies followed for deriving PDF transport equations can be found in $[2,3,5]$.

\subsection{Lagrangian Particle Equations}

As it can be observed from the corresponding expressions shown in the previous section, PDF transport equations have a large number of dimensions. This high dimensionality is directly related to the number of scalars (chemical species plus enthalpy) transported, which can be of the order of tens and even hundreds when detailed chemical mechanisms are accounted for. These equations are seldom solved using conventional finite volume or finite difference methods. Monte Carlo techniques are often used for these purposes. In Lagrangian Monte Carlo simulations the computational domain is usually discretized (in physical space) into a number of cells in order to extract local mean quantities that characterize the evolution of particles. Within each cell at any given time $t$, the joint PDFs are represented by an ensemble of stochastic particles [24], whose evolution yields the same one-point, one-time Eulerian PDF as the real fluid particle system [2]. Solving therefore for the evolution of these particles effectively corresponds to solving a modeled PDF transport equation.

Particles are in general characterized by their position, velocity and composition, which at time $t$ can be denoted by $x^{*}(t), u^{*}(t), \phi^{*}(t)$, respectively. Additionally a particle property including turbulence timescale information, i.e., turbulence frequency $\omega^{*}(t)$, can also be considered. The stochastic particle system is governed by a set of stochastic (ordinary) differential equations (SDEs). Accordingly the evolution of the $i^{\text {th }}$ stochastic particle properties can be written as (following a generic representation similar to that used in [24]),

$$
\begin{aligned}
& \frac{d x^{*,(i)}}{d t}=u^{*,(i),} \\
& \frac{d u^{*,(i)}}{d t}=A^{*,(i)},
\end{aligned}
$$




$$
\begin{gathered}
\frac{d \phi_{\alpha}^{*,(i)}}{d t}=\Phi_{\alpha}^{*,(i)}+S_{\alpha}\left(\phi^{*,(i)}\right) \\
\frac{d \omega^{*,(i)}}{d t}=\Theta^{*,(i)}
\end{gathered}
$$

where $A^{*,(i)}$ represents a model for the particle acceleration, $\Phi_{\alpha}^{*,(i)}$ is the mixing model, $S_{\alpha}\left(\phi^{*,(i)}\right)$ the reaction rate corresponding to the $\alpha^{\text {th }}$ scalar, and $\Theta^{*,(i)}$ is a model representation for the turbulence frequency. It is worth noticing that Eqs. (16)-(19) correspond to a generic representation of the set of SDEs utilized. As it will be shown later on in this section then, the models referred to in these equations include in general both closed and open terms. Accordingly, once the stochastic particle system aims to represent the fluid system, the Fokker-Planck equation [34] describing the transport of the joint PDF of $x^{*}(t), u^{*}(t), \phi^{*}(t)$ and $\omega^{*}(t)$ is made to correspond with the PDF of SGS fields [28]. The implied closure for the SDEs, Eqs. (16)-(19), is then obtained by comparing this Fokker-Planck equation with the exact joint PDF transport equations described in the previous section. As a result different sets of SDEs, involving different models as well, are solved for different joint PDF transport equations.

For joint frequency-velocity-composition PDFs, for instance, a set of SDEs that has been utilized involves [28],

$$
\begin{gathered}
d x_{i}^{*,(i)}=u_{i}^{*,(i)} d t+\sqrt{\frac{2 \mu}{\langle\rho\rangle_{l}}} d W_{i}^{(i)} \\
d u_{i}^{*,(i)} \\
=\left\{\begin{array}{c}
\left.-\frac{1}{\langle\rho\rangle_{l}} \frac{\partial\langle p\rangle_{l}}{\partial x_{i}}+\frac{2}{\langle\rho\rangle_{l}} \frac{\partial}{\partial x_{j}}\left(\mu \frac{\partial\left\langle u_{i}\right\rangle_{L}}{\partial x_{j}}\right)+\frac{1}{\langle\rho\rangle_{l}} \frac{\partial}{\partial x_{j}}\left(\mu \frac{\partial\left\langle u_{j}\right\rangle_{L}}{\partial x_{i}}\right)\right) d t \\
+\frac{2}{3} \frac{1}{\langle\rho\rangle_{l}} \frac{\partial}{\partial x_{i}}\left(\mu \frac{\partial\left\langle u_{j}\right\rangle_{L}}{\partial x_{j}}\right) \\
+G_{i j}\left(u_{j}^{*,(i)}-\left\langle u_{j}\right\rangle_{L}\right) d t+\sqrt{C_{0} k \Omega} d W_{i}^{\prime,(i)}+\sqrt{\frac{2 \mu}{\langle\rho\rangle_{l}}} \frac{\partial\left\langle u_{i}\right\rangle_{L}}{\partial x_{j}} d W_{j}^{(i)} \\
d \phi_{\alpha}^{*,(i)}=-C_{\phi} \Omega\left(\phi_{\alpha}^{*,(i)}-\left\langle\phi_{\alpha}\right\rangle_{L}\right) d t+S_{\alpha}\left(\phi^{*,(i)}\right) d t
\end{array}\right.
\end{gathered}
$$




$$
d \omega^{*,(i)}=-C_{\omega} \Omega\left(\omega^{*,(i)}-\widehat{\omega}\right) d t
$$

where $W$ terms represent Wiener-Lévy processes [35] and $k$ and $\Omega$ are, respectively, the SGS kinetic energy and mixing frequency. In these SDE equations, particle velocities have been modeled using the simplified Langevin model (SLM) [3,36], whereas the interchange by exchange with the mean [37] model is utilized for modeling the mixing process. In SLM $G_{i j}$ is modeled as a function of the SGS mixing frequency $\Omega . C_{0}, C_{\phi}$ and $C_{\omega}$ are in turn model parameters. Notice that the frequency model, Eq. (23), involves here a relaxation equation that causes the stochastic frequency to relax to the local frequency $\widehat{\omega}$ evaluated on the grid points [28].

Except for the last equation dealing with the modeling of turbulence frequency, the same set of SDEs described by Eqs. (20)-(23) have been used for the case of joint velocity-composition PDFs [32]. The only difference is that no frequency model is utilized in this case. Information on flow scales needs thus to be obtained here from additional ad hoc models.

A further reduced set of SDEs is associated with joint composition PDF formulations. In these particular situations, the expression corresponding to the transport of the PDF in composition space, Eq. (22), as it is, together with an equation governing the trajectory of particles in physical space of the form [33],

$$
d x_{i}^{*,(i)}=\left[\left\langle u_{i}\right\rangle_{L}+\frac{\partial\left(\Gamma+\Gamma_{t}\right)}{\partial x_{i}}\right] d t+\left[2\left(\Gamma+\Gamma_{t}\right)\right]^{1 / 2} d W_{i}^{(i)},
$$

complete the set of SDEs required for this case. In Eq. (24) $\Gamma_{t}$ represents the turbulent diffusion coefficient. Notice that in these sets of SDEs, the PDF transport due to molecular diffusion has been modeled by (i) a random walk in physical space (last RHS terms of Eqs. (20) and (24)), and (ii) a mixing process (first RHS term of Eq. (22)). It has been argued that this approach gives rise to spurious production of scalar variance that does not vanish as the LES filter width becomes small [38]. Modifications proposed to correct this issue involve modeling the PDF spatial diffusive transport by a mean drift term in the scalar evolution equation (Eq. (22)) [39].

All three types of joint PDFs usually transported, i.e., frequency-velocitycomposition, velocity-composition and composition PDFs, require models for scalar mixing, $\Phi_{\alpha}^{*,(i)}$, because the corresponding transport equations are formulated for one- 
point, one-time statistics. One way of avoiding these mixing models would involve the use of multiple-point, multiple-time statistics. Nevertheless this is not commonly done in practice due to the complexity involved when using these formulations. For details about multi-point descriptions, see for example references [4,40-42].

Finally it is worth highlighting that joint frequency-velocity-composition PDF formulations are sometimes referred as self-contained PDF methods. This occurs because the corresponding PDFs include turbulence timescale information that allows forming a complete turbulence model. The closed system thus formed requires no further information except for fluid properties and initial and boundary conditions [43]. Although they can initially appear very attractive, self-contained methods require a relatively large number of particles per computational cell [44], thus limiting their use for the simulation of complex turbulent reacting flows. Hybrid Euler/Lagrange approaches requiring fewer particles per cell have been preferred for modeling this type of flows. Hybrid methods seek to take advantage of the strengths of particle-based and grid-based procedures, which include, respectively, exact treatments of advection in physical space and of one-point source terms in PDF transport equations [45].

\section{State-of-the-art of Mixing Models}

The single most important, and probably the weakest, element in hybrid Euler/Lagrange approaches - used for the simulation of turbulent reacting flows - is the modeling of the term that physically represents the transport of the PDF by the action of molecular diffusivity [2]. It is worth highlighting here that there are two different physical effects that may be distinguished, (i) transport in physical space and (ii) transport ('mixing') in composition space [38,39]. It has been said that the lack of a general and accurate mixing model is a major source for uncertainties in turbulent combustion simulations [46]. Hence the great importance of developing accurate mixing models able to adequately model the interactions between turbulence and chemical reaction processes.

There are several requirements that mixing models must fulfill in order to yield the results expected from these models. One of the foremost prerequisites associated with these models relates to their physically based simplicity, as they should both reflect a physical phenomenon and be simple enough to allow their use in practical applications. 
Several other requirements for mixing models, coming essentially from the nature of molecular diffusion, are discussed in literature $[4,47,48]$. The most essential of these imply that [2]: (i) mean scalar quantities should not change as a result of mixing, (ii) scalar variances should decay at the correct rate (i.e., scalar dissipation rates should be correct), and (iii) scalar quantities should remain bounded or in the allowable region (e.g., mass fractions should remain between zero and unity and sum to unity). In addition to these three requirements, there are others [2,4,47-48] involving the fact that, for example, mixing models should have characteristics of linearity and independence, the mixing process should be local in composition space, the PDF of conserved scalars should relax to a Gaussian distribution in statistically homogeneous systems, and mixing models should include dependencies on Reynolds, Schmidt and Damköhler numbers.

In addition, for velocity-composition PDF approaches, it has been argued that scalar mixing models should include an explicit dependence on velocity [49]. Indeed the effect of neglecting velocity-conditioning in mixing models has been investigated previously [50,51] and has been found to be significant. Most mixing models do not consider differential diffusion (the phenomenon in which different scalars with different molecular diffusivities evolve differently from each other [52]), neither a direct influence on mixing of turbulence length scales and chemical reactions [2]. It is worth noticing that most mixing models have been initially developed for constant density turbulence and later on applied to variable density reacting flows. Significant thermal expansion occurring in the latter case might cause large differences on how scalar gradients or, equivalently, scalar fluctuation dissipation rates are enhanced or destroyed. So the presence of chemical reactions needs to be accounted for if mixing models for turbulent combustion resting on sound physical grounds are to be developed. Although much effort has been devoted to the development of mixing models that improve upon the existing ones, no mixing model completely satisfies all of the desirable properties indicated above $[4,48]$. In general all current models have certain shortcomings with respect to these requirements. The main reason for this is that, generally speaking, most mixing models are based on simple physical arguments. Indeed most often only vague mechanistic notions of the turbulent mixing process and some guiding rules have been used in mixing model development. It seems that this has been a rather unproductive way of operating, with limited improvements and understanding after 50 years of 
efforts. If more accurate mixing models are to be developed then, a better physical understanding of the molecular mixing process is mandatory.

Particle-based mixing models for turbulent combustion originate in the work of Flagan and Appleton [53], who developed a stochastic model of turbulent mixing where mixing was represented by random fluid particle interactions. Most mixing models have been developed for RANS (Reynolds-averaged Navier Stokes) based simulations, i.e., in a RAS context. In this work mixing models are then reviewed in this context. Nevertheless it is worth highlighting that, with the advance of computational power, hybrid Euler/Lagrange approaches based on LES/FDF formulations are being preferred nowadays for the modeling of turbulent reactive flows. Since FDF and PDF transport equations have a similar structure, they have been solved with similar stochastic particle methods. Desirable characteristics of diffusion models used in LES/FDF contexts are thus similar to those discussed above corresponding to RAS/PDF ones [38]. Accordingly the same mixing models have been utilized in both cases $[16,17,54]$. Nevertheless, as highlighted in [55], there are some open questions about the performance of mixing models in LES/FDF calculations. These questions include the effect of both small scales and molecular diffusion on the mixing processes, the optimal value of the scalar-to-mechanical timescale ratio, and the proper modeling of the turbulent mixing process [55].

One key aspect to emphasize relates to fact that in LES/FDF contexts the model formulation must reduce to a Direct Numerical Simulation (DNS) in the limit of vanishing filter width (DNS limit [38]). Additionally it has been observed from numerical studies that LES/FDF results tend to be less sensitive to the details of mixing models compared to RAS/PDF results [56]. This agrees with the argument that relatively simple mixing models (dealing with SGS fluctuations only) should suffice for LES/FDF, in contrast to RAS/PDF where mixing models must represent the effects of all fluctuations [2]. This is one of the reasons why, when utilized in RAS contexts, early mixing models do not perform well for high Damköhler numbers where molecular transport and chemical reactions are tightly coupled [2]. As it will be discussed in the following sections, this problem of high Damköhler number has been recognized for years and several approaches have been proposed to address it. The way in which the composition of a particle changes, i.e., (i) essentially independently of that of the other particles or (ii) through direct interaction with other particles [2], defines two broad 
categories of particle-based scalar mixing models. A review of some of the most widely known mixing models used in hybrid Euler/Lagrange formulations and belonging to both categories is presented below.

\subsubsection{Interaction by Exchange with the Mean (IEM)}

The Interaction by Exchange with the Mean (IEM) model [37], also known as Linear Mean-Square Estimation (LMSE) model [57], is the simplest model belonging to the category of models where the composition of a particle changes essentially independently of the corresponding ones associated with the other particles. Because of the simplicity of its numerical implementation, this mixing model is the most widely used in hybrid Euler/Lagrange simulations. As originally proposed, when using the IEM model, the scalar values of the $i^{\text {th }}$ particle evolve according to,

$$
\frac{d \phi^{(i)}}{d t}=-\frac{1}{2} \frac{C_{\phi}}{\tau}\left(\phi^{(i)}-\left\langle\phi \mid x^{(i)}\right\rangle\right)
$$

where $C_{\phi}$ is a model parameter (known as scalar-to-mechanical time scale ratio), $\tau$ the turbulence time scale (inverse of the mean turbulence frequency), and $\langle\phi \mid x\rangle$ is the mean of $\phi$ conditional upon $x$. For the sake of clarity the superscript ${ }^{*}$ denoting particles is dropped in this section. Notice that in Eq. (25) it has been assumed that the mixing time scale, $\tau_{\phi}$, is proportional to the turbulence time scale,

$$
\tau_{\phi}=\frac{\tau}{C_{\phi}}
$$

with proportionality constant equal to the model parameter $C_{\phi}$ [5]. This parameter cannot be regarded as a universal constant. Accordingly different values have been reported in literature [2,58]. Usually different mixing models require different $C_{\phi}$ values as well, so choosing an optimal value for $C_{\phi}$ is an ad hoc process and depends on the particular mixing model utilized [59]. Indeed $C_{\phi}$ is expected to vary with the characteristic length scales of the scalar fields considered [60]. More sophisticated models for the mixing time scale have been also reported in previous works $[4,59]$.

The IEM model possesses the three most essential characteristics of a mixing model indicated above. However, in homogenous turbulence, due to the absence of mean scalar gradients, IEM preserves the shape of the scalar PDF, which never relaxes 
[4]. When used as originally proposed - Eq. (25) - in joint velocity-composition PDF calculations, the IEM model presents another limitation, as it does not include an explicit dependence on velocity. In other words, as most scalar mixing models, it assumes that molecular mixing is independent of the instantaneous velocity. However, since in joint velocity-composition PDFs the velocity is known, this assumption is unnecessary and leads to a lack of local isotropy in the scalar field [61]. IEM variants that include a dependence on velocity have been proposed in the past as follows. The problem of lack of local isotropy was noted by Pope [49] through high Reynolds (Re) number analyses, using turbulent dispersion theory, of the scalar flux evolution that is implied by the IEM model. This led to an IEM extension, known as Interaction by Exchange with the Conditional Mean (IECM) model,

$$
\frac{d \phi^{(i)}}{d t}=-\frac{1}{2} \frac{C_{\phi}}{\tau}\left(\phi^{(i)}-\left\langle\phi \mid u^{(i)}, x^{(i)}\right\rangle\right),
$$

where the relaxation is towards the mean conditioned on velocity as well as on position (rather than on position only).

Even though local isotropy should prevail as $\operatorname{Re} \rightarrow \infty$, at moderate Re numbers local anisotropy may be significant [61]. In order to account for this aspect, Fox [61] introduced a local anisotropy parameter $\varsigma$ giving rise to the so called velocityconditioned IEM (VCIEM) model,

$$
\frac{d \phi^{(i)}}{d t}=-\frac{1}{2} \frac{C_{\phi}}{\tau}\left[\phi^{(i)}-(\varsigma)\left\langle\phi \mid x^{(i)}\right\rangle-(1-\varsigma)\left\langle\phi \mid u^{(i)}, x^{(i)}\right\rangle\right]
$$

where $\varsigma=0$ corresponds to local isotropy and $0<\varsigma \leq 1$ represents the degree of local anisotropy. The local anisotropy parameter exhibits a Reynolds number dependence that is determined using experimental or DNS data.

Other IEM extensions include those based on Fokker-Planck equations for joint velocity-composition PDF and FDF formulations, e.g., Refined Interaction by Exchange with the Mean (RIEM) [62,63] and Stochastic Interaction by Exchange with the Mean (SIEM) models [60]. In the Extended IEM (EIEM) model proposed by Sabel'nikov and co-workers [64-66], in order to account for a turbulent frequency scale distribution, the single mean scalar frequency used in the original IEM model is replaced by a distribution of scalar frequencies. This representation of a whole distribution of scales is interpreted as fluid particles possessing their own frequency and behaving as if 
belonging to different structures [65]. Both classical [64] and modified [65] log-normal laws have been used for characterizing the stochastic process evolution associated with the distribution of scalar frequencies utilized. EIEM allows for the scalar PDF to relax towards a Gaussian distribution in statistically homogeneous systems. Analytical solutions exist for this model in simplified situations [67]. In general the IEM model assumes a linear relaxation of the scalars towards their mean value. Moreover, as given in Eq. (25)-(28), it implies that all scalars mix at the same rate so differential diffusion is not accounted for in the model [4]. Another significant IEM limitation relates to fact that it is non local in composition space.

\subsubsection{Coalescence and Dispersion (CD)}

Curl's Coalescence and Dispersion (CD) model [68] represents the simplest model belonging to the group of models where the composition of a particle changes through direct interaction with other particles. This model, which served later on as inspiration for the works of Janicka et al. [22] and Dopazo [69] dealing with turbulent mixing phenomena, was originally intended to describe droplets interactions in a two-liquid phase chemical reactor. Its name is fully adequate then when describing droplet interactions (Curl's original application), but it could be misleading when used for gaseous systems. Even so in this work the coalescence-dispersion denomination is used for referring to this mixing model since it is the most frequently used form found in the literature. In this model, in each mixing event, pairs of particles are randomly selected and their resulting individual concentrations are identical and equal to their average value (coalescence-dispersion process). A mass conservative generalization of Curl's formulation, for unequally weighted particles, can be expressed as [24,25],

$$
\phi^{(i), t+1}=\phi^{(j), t+1}=\phi_{c}, \quad \phi_{c}=\frac{w^{(i)} \phi^{(i), t}+w^{(j)} \phi^{(j), t}}{w^{(i)}+w^{(j)}} .
$$

More specifically Eq. (29) highlights the scalar values of a pair of mixing particles $i$ and $j$ after a given mixing event or time step $(t \rightarrow t+1)$.

In the $\mathrm{CD}$ model the mixing rate is controlled by the fraction of participating particle pairs that is given by the total weight of all particles considered during the mixing process [25]. Similarly to the IEM model, CD possesses the three most essential characteristics required in a mixing model. However, in contrast to IEM, CD does not 
preserve the shape of the scalar PDF and neither relaxes towards a Gaussian distribution in statistically homogeneous systems [2].

Several modifications have been introduced to the original CD model over the years in order to remediate some of its main deficiencies. One of the early modifications corresponds to the Modified Coalescence and Dispersion (MCD) model proposed by Janicka et al. [22]. In contrast to the results obtained from Curl's model, MCD yields continuous PDF's. This is achieved by introducing an additional random parameter $h$ with a continuous PDF that controls the extent of mixing on each mixing event,

$$
\begin{aligned}
& \phi^{(i), t+1}=\phi^{(i), t}+h\left(\phi_{c}-\phi^{(i), t}\right), \\
& \phi^{(j), t+1}=\phi^{(j), t}+h\left(\phi_{c}-\phi^{(j), t}\right) .
\end{aligned}
$$

Here $h$ varies between 0 and 1 so when $h=0$ no mixing occurs, and for $h=1$ Curl's model is recovered. Although MCD is successful in producing a continuous PDF, it does not relax towards a Gaussian distribution. An attempt in this direction is the model described in [70], where the selection of particles for mixing is biased. The bias utilized depends upon the age of the particle, which is defined as the time since it last participated in a mixing event. Results obtained with age biasing show PDF distributions that relax to near-Gaussian shapes for passive scalars in homogeneous turbulence [70].

The most significant limitation of Curl's model and variants (involving random selections of particles for mixing) is that they are non local in composition space. This occurs mainly because of the random selection procedure utilized, which yields discontinuous jumps in particle compositions that violate the localness requirement. Since mixing models are used for mimicking molecular diffusion, a continuous process in space and time, jumping in composition space over an infinitesimal time interval is inherently unphysical [4]. This aspect may be critical in combustion regimes where the reaction rate is significant over a thin region in composition space only. In these situations such models could allow fluid particles to mix across the reaction zone without burning [2]. This is one of main reasons why these models are not preferred nowadays for transported PDF simulations of reacting flows [4]. It is worth noticing however that, over a finite time interval, physical fields could make jumps in composition space. This means that a priori jumps in composition space need not be 
unphysical. The issue with mixing models here is how to handle the integrated effect of such jumps, in terms of scalar values, over a finite time interval.

\subsubsection{Mapping Closure (MC)}

The Mapping Closure (MC) model [20] is a particle implementation of the model described by Chen et al. [71] involving Gaussian reference fields. MC is based on the use of three elements: (i) a specified statistically homogeneous, isotropic, timeindependent Gaussian field, where all multipoint statistics are known in terms of its mean, variance and two-point correlation function; (ii) a surrogate field, whose known statistics are the same as those associated with the stochastic field of interest; and (iii) a mapping, which is used to map the Gaussian reference field to the surrogate one. The condition of uniqueness of the mapping used in $\mathrm{MC}$ requires that particles are first sorted in non decreasing order of composition values [24]. This process of ordering of scalars leads to some difficulties when the MC model is to be extended for multi scalar mixing applications.

Given an ensemble of single-scalar $N_{p}$ particles, the evolution of the scalar values according to MC proceeds as follows (after a generalization for unequally weighted particles) [25],

$$
\frac{d \phi^{(i)}}{d t}=\frac{1}{2} \frac{C_{\phi}^{\prime}}{\tau}\left[B_{i+1 / 2}\left(\phi^{(i+1)}-\phi^{(i)}\right)-B_{i-1 / 2}\left(\phi^{(i)}-\phi^{(i-1)}\right)\right]
$$

where $C_{\phi}^{\prime}$ is a model constant. In this model each particle evolves by interaction with its

neighbors in composition space with a rate of attraction, $B_{i \pm 1 / 2}$, equal to $\left(B_{1 / 2}=\right.$ $\left.B_{N_{p}+1 / 2}=0\right)$,

$$
B_{i+1 / 2}=\frac{N_{p} g\left(\eta_{i+1 / 2}\right)}{\eta_{i+1}-\eta_{i}}, \quad i=1,3, \ldots, N_{p}-1
$$

In this last expression, $g(\eta)$ represents the standard Gaussian density function and $\eta$ the sample space coordinate, which is related to particle weights through the Cumulative Density Function (CDF) corresponding to $g$.

This model is local in composition space because only neighbor particles are considered during the mixing processes. It also possesses the three most essential features required in mixing models, i.e., it satisfies mean conservation, variance decay 
and the boundedness principle. One advantage of MC-based models is that the PDF of conserved scalars relax towards a Gaussian distribution in statistically homogeneous systems [2]. It should be stressed though that problems arise when the MC model is extended to multiple reactive scalars, owing to mappings that are non unique and expensive to compute [24]. This and other issues have been addressed in the formulations based on mapping closures developed more recently [24].

\subsubsection{Euclidean Minimum Spanning Tree (EMST)}

Since IEM and CD models are non local in composition space they do not perform well for high Damköhler numbers [2]. One of the early approaches proposed for solving this problem involves the ordered pairing model [21]. In this model, in order to respect the localness principle, particles selected for mixing are firstly ordered. Because of the nature of the problem analyzed (turbulent diffusion flame in flamelet regime), the particle ordering process is carried out according to their mixture fraction. Particle mixing is then performed by using an adjacent pairing scheme where adjacent order particles are selected as mixing partners. Although this model yields good results in terms of locality, the mixture fraction PDF does not approach the desired Gaussian distribution. Because of the ordering process utilized, the ordered pairing model resembles the MC model. This procedure is carried out in both models such that the results are in better agreement with the notion of locality under discussion.

A more general approach involving a bias for selecting particle pairs for mixing in such a way that particles closer in composition space are more likely to mix than those further away - constitutes the Euclidean Minimum Spanning Tree (EMST) [24] model. EMST is an extension of the MC model [20] for multiple scalars, where the onedimensional ordering in scalar space is replaced by an Euclidean minimum spanning tree [25]. In one-dimensional mapping closures for instance, each particle has two neighbors, except those particles located at the ordering list extremes, which have one only. Similarly, in the EMST model each particle has at least two spanning tree edges incident on it, except those at the extremes. Edge weights are defined as a function of particle weights, such that they have minimum values at the extremes of the distribution and increase as they approach the median. 
Specifically, for an ensemble of $N_{p}$ particles $\phi_{\alpha}^{(i)}$ with a $\left(N_{s}+1\right)$-dimensional composition space $\left(\alpha=1,2, \ldots, N_{s}+1\right)$, the matrix form of the EMST model evolution is expressed as [24],

$$
\frac{d \phi_{\alpha}^{(i)}}{d t}=-\frac{1}{w^{(i)}} M_{i j} \phi_{\alpha}^{(j)}, \quad i=1, \ldots, N_{p}^{\prime}\left(N_{p}^{\prime} \leq N_{p}\right)
$$

where the elements of the interaction matrix $M_{i j}$ are given by

$$
\begin{gathered}
M_{i j}=-\alpha_{p} \sum_{v=1}^{N_{p}^{\prime}-1} B_{v}\left(\delta_{i m_{v}} \delta_{j n_{v}}+\delta_{j m_{v}} \delta_{i n_{v}}\right), \quad i \neq j \\
M_{(i)(i)}=\alpha_{p} \sum_{j=1}^{N_{p}^{\prime}} \sum_{v=1}^{N_{p}^{\prime}-1} B_{v}\left(\delta_{i m_{v}} \delta_{j n_{v}}+\delta_{j m_{v}} \delta_{i n_{v}}\right) .
\end{gathered}
$$

In these last two expressions, $B_{v}$ represents the edge coefficient associated with the $v^{\text {th }}$ spanning tree edge connecting particles $m_{v}$ and $n_{v}$. This coefficient is defined as a linear function of the edge weight, which is directly related to the weights of the particles that belong to the two subtrees resulting from the removal of edge $v$ from the set of EMST edges. $\alpha_{p}$ is in turn a model parameter controlling the rate of variance decay of the scalars, and $\delta$ represents the Kronecker delta. Notice that at any given time only a subset, $i=1, \ldots, N_{p}^{\prime}$, of the total ensemble of particles $N_{p}$ participates in the mixing process. Particles belonging to the mixing subset are determined by a non dimensional particle age property, which determines how long a particle mixes and or not. The time a particle spends in the mixing/non mixing states is required to correspond with the turbulence timescale in the grid cell.

EMST has been developed focusing on localness preservation. As such it is local in composition space and possesses the three most essential characteristics of a mixing model. It does not fully satisfy however the linearity and independence properties $[2,24]$. In addition, the lack of information concerning the joint scalar dissipation rates in the EMST model can also lead to spurious results, which implies that while localness is a desirable property, it is not sufficient for ensuring physically realistic predictions [4]. The description of other EMST shortcomings can be found in [72]. 


\subsubsection{Multiple Mapping Conditioning (MMC)}

A different generalization of the mapping closure concept constitutes the Multiple Mapping Conditioning (MMC) model [73]. Although usually referred to as a model, MMC is in fact an entire framework where different specific models can be formulated $[25,73,74]$. This methodology for mixing and reaction effectively unifies the joint PDF [2,5] and Conditional Moment Closure (CMC) [75] approaches in such a way as to account for their advantages. When MMC is formulated stochastically it becomes a PDF model where mixing is local or conditioned within a reference space [74]. MMC as a PDF method is similar to the EMST model [24] because both models use the MC concept and search the neighborhood of particles to define their interactions [76]. In contrast to EMST, however, MMC uses one or more reference variables to enforce locality in composition space.

The original MMC model [73] requires the reference variables to be modeled by Markov processes. This restriction has been removed in a more general understanding of MMC [76], so that reference variables currently may be other known quantities, including data interpolated from Eulerian LES or DNS results [74]. In terms of mixing, MMC effectively unites many existing models [77], including IEM [37] or VCIEM [61], CD [68] and CMC [75]. For instance, using a two-particle interaction Curl-like model, at each time step the mixing of a pair of particles, $p$ and $q$, proceeds according to $[74,76]$

$$
\begin{aligned}
& \phi^{(p), t+1}=\phi^{(p), t}+\alpha_{p}\left(\hat{\phi}^{(p, q)}-\phi^{(p), t}\right), \\
& \phi^{(q), t+1}=\phi^{(q), t}+\alpha_{p}\left(\hat{\phi}^{(p, q)}-\phi^{(q), t}\right),
\end{aligned}
$$

where $\hat{\phi}^{(p, q)}$ is the weighted two-particle average, and $\alpha_{p}$ the mixing extent $\left(\alpha_{p} \in\right.$ $[0,1])$. In MMC localness is preserved by enforcing mixing interactions of particles that are close in both reference and physical space. Thus particles that are close in both spaces are allowed to be mixed only. The selection of particle pairs is thus an important step in the mixing process. Pairs of particles (e.g., $p$ and $q$ ) are selected such as to minimize the normalized square distance given by $[74,76]$ 


$$
\hat{d}_{(p, q)}^{2}=\frac{1}{1+\lambda^{2}}\left[\sum_{j=1}^{3}\left(\frac{x_{j}^{(p)}-x_{j}^{(q)}}{L_{x}}\right)^{2}+\lambda^{2}\left(\frac{\tilde{f}^{(p)}-\tilde{f}^{(q)}}{L_{f}}\right)^{2}\right]
$$

In Eq. (39) $L_{x}$ and $L_{f}$ are characteristic physical and reference scales, and $\lambda$ is a parameter determining, respectively, the relative localization in physical and reference spaces. $x$ and $\tilde{f}$ represent in turn particle physical and reference locations, respectively. Notice that, depending on the values of $\lambda$, localization can be in physical space only $(0)$ or in reference space only $(\infty)$. MMC possesses the three most essential features expected of a mixing model [2]. Particularly, in this model, locality in composition space is achieved, boundedness of scalars is preserved, linearity and independence principles are also fulfilled, and when required the resulting PDF tends to a Gaussian distribution [73].

Recent work developed about MMC [74,76] describes a novel numerical method for solving PDF transport equations with MMC closures, which is referred to as sparseLagrangian simulations. In this type of simulations fewer Lagrangian particles than Eulerian cells are utilized, in contrast to the commonly used intensive-Lagrangian simulations employing many Lagrangian particles per Eulerian cell. The use of sparseLagrangian schemes greatly reduces, as expected, the computational time associated with the simulations of turbulent reacting flows. For instance, a simulation of a partially premixed jet diffusion flame (Sandia Flame D), using realistic chemical kinetics containing 34 reactive species and 219 reactions, took about 50 hours on a single dualcore workstation, only [76].

It is worth noticing that the rigorous consistency and tight two-way coupling between Eulerian and Lagrangian representations, usually required in hybrid intensiveLagrangian simulations, has been relaxed in the sparse-Lagrangian ones. In addition, the filtered density field at the grid scale cannot be extracted using this last approach [2]. Likewise intensive approaches [16,78,79], in sparse formulations, Eulerian/Lagrangian density coupling is achieved by solving an Eulerian transport equation for equivalent enthalpy. However, the source term of this equation is modeled, in contrast to intensive simulations, where it may be obtained from particle properties. This source term modeling involves the evaluation of conditional means calculated from an ensemble of particles in the near-neighborhood of a given position [80]. 


\subsubsection{Shadow-Position Mixing Model (SPMM)}

One of the most recent efforts in the development of mixing models is the Shadow Position Mixing Model (SPMM) [72]. This model has been constructed in such a way that it is consistent with turbulent dispersion theory and local in composition space. Mixing is modeled as a relaxation of the composition to its mean conditioned on a new variable, the shadow position $Z$. This new variable corresponds to the position of a "shadow" particle associated with each of the Lagrangian particles transported. The evolution of the shadow position $Z$ proceeds according to a SDE of the form

$$
d Z^{(i)}=\left\langle u^{(i)} \mid x^{(i)}\right\rangle d t-\frac{a_{m}}{T_{L}}\left(Z^{(i)}-x^{(i)}\right) d t+b_{m}\left(2 \sigma^{2} T_{L}\right)^{1 / 2} d W^{(i)},
$$

which includes terms related to its movement due to the local mean velocity, relaxation towards the Lagrangian particle position and a random walk (Wiener process). In Eq. (40) $T_{L}$ is the Lagrangian integral time scale proportional to the ratio of turbulence kinetic energy and mean dissipation rate, $a_{m}$ and $b_{m}$ are positive model coefficients and $\sigma$ is the root mean square velocity. Expressed in terms of the so called shadow displacement $R$, defined as $R(t) \equiv Z(t)-x(t)$, whose SDE is given by (from Eqs. (16) and (40))

$$
d R^{(i)}=\frac{a_{m}}{T_{L}} R^{(i)} d t-\left(u^{(i)}-\left\langle u^{(i)} \mid x^{(i)}\right\rangle\right) d t+b_{m}\left(2 \sigma^{2} T_{L}\right)^{1 / 2} d W^{(i)},
$$

the composition in the SPMM evolves according to

$$
\frac{d \phi^{(i)}}{d t}=-\frac{C_{\phi}}{T_{L}}\left(\phi^{(i)}-\left\langle\phi \mid R^{(i)}, x^{(i)}\right\rangle\right),
$$

where $C_{\phi}$ is a model constant.

SPMM possesses the three most essential characteristics associated with a mixing model. It has been constructed to be consistent with turbulent dispersion theory and local in composition space, as only conditioning space neighbor particles are allowed to mix. Additionally, it satisfies linearity and independence principles, as well as it yields Gaussian PDF distributions in statistically homogeneous systems [72]. It has been shown that, when applied to a turbulent non premixed flame (reactive scalar mixing layer configuration), SPMM correctly yields stable combustion, in contrast to both IEM [37] and IECM [49] models which incorrectly lead to extinction under the same 
circumstances. Because of the use of conditioning variables, SPMM involves some of the ideas of MMC [73] modeling. Moreover for different limiting values of its coefficients, SPMM reduces to IEM, IECM or MMC mixing models.

In numerical terms, SPMM uses a mesh free, near neighbor implementation. As such, at each time step, computational particles are ordered so adjacent particles in the ordering are near neighbors in the conditioning space $(x, R)$. Even though the use of other conditioning variables adds more complexities, SPMM has been extended - e.g., Velocity Shadow Position Mixing Model (VSPMM) - such as conditioning is performed on particle velocity as well. The advantage of VSPMM is that it exactly satisfies dispersion consistency, and can satisfy localness, either exactly or approximately, according to the values of the model coefficients characterizing the VSPMM.

\subsubsection{Parameterized Scalar Profile (PSP)}

A simple interpretation of the IEM mixing model [37] points out that its use implies that scalars evolve as if all fluid particles were located on one dimensional sinusoidal scalar profiles with both same length scale and same profile center value. The Parameterized Scalar Profile (PSP) [46] mixing model involves then a general parameterization of these one dimensional scalar profiles; i.e., instead of using the same length scales and center values for all profiles, these parameters are modeled. Considering that the scalar profiles have a sinusoidal shape, the profile parameterization is carried out as follows. For the $i^{\text {th }}$ particle its associated one dimensional scalar profile is characterized by a minimum value $\phi_{-}^{(i)}$, a maximum value $\phi_{+}^{(i)}$ and a length scale $\lambda^{(i)}$. According to PSP, the scalar evolution is then given by [46]

$$
\frac{d \phi^{(i)}}{d t}=-\frac{1}{\tau^{(i)}}\left(\phi^{(i)}-\phi_{c}^{(i)}\right)
$$

where

$$
\tau^{(i)}=\frac{2}{C_{\phi} \omega^{(i)}}, \quad \phi_{c}^{(i)}=\frac{\left(\phi_{-}^{(i)}-\phi_{+}^{(i)}\right)}{2}, \quad \lambda^{(i)}=\pi \sqrt{\frac{2 \Gamma}{C_{\phi} \omega^{(i)}}}
$$

In Eq. (44) $\omega$ represents the turbulence frequency, $\Gamma$ is the diffusion coefficient and $C_{\phi}$ a model constant. The turbulence frequency, which is directly related to the profile length 
scale $\lambda$, evolves according to a SDE such as the gamma distribution-based one highlighted in [81].

Profile (minimum and maximum) boundaries are represented by the scalars of other two particles belonging to the same cell where the $i^{\text {th }}$ particle is located. In addition the following condition

$$
\left(\phi_{-}^{(i)}-\phi^{(i)}\right)\left(\phi_{+}^{(i)}-\phi^{(i)}\right) \leq 0
$$

is enforced. Profiles are considered degenerated as soon this condition is violated. In this case new representatives for the profile boundaries are randomly selected. In order to account for spatial decorrelation of particle triplets (consisting of the on-profile particle and the two particles representing the profile boundaries) with time, profile boundaries are also reinitialized using an intermittent aging process. Each particle carries two decreasing time stamps, $t_{-}^{(i)}$ and $t_{+}^{(i)}$, for this purpose. There are certain similarities between the PSP and the Fokker-Planck (FP)-based model proposed by Fox [82]. Compared to this model, some of the differences of PSP relate to the use of stochastic processes for the evolution of the profile boundaries and length scale. PSP possesses the three essential features required in mixing models, although boundedness in multi scalar formulations may be weakly violated. Linearity and independence principles are fulfilled approximately only, and the model is non local in composition space.

Several extensions of the original PSP mixing model have been proposed over the years. Two of which, for inhomogeneous mixing, relate to (i) multiple computational grid cells and (ii) unequally weighted particles [25]. In order to replace profile boundary representatives, which no longer belong to the same grid cell as the on-profile particle, a deterministic IEM-like model has been used to evolve profile boundary values. Particles weights have also been used as proportionality constants for selecting particles at the beginning of profile-boundary life cycles. PSP has also been extended in such a way that it provides joint scalar-scalar dissipation rate PDFs [83] and better reproduces the behavior of fluid particles [48]. Profile boundary representatives, or drift compositions, have been more recently modeled using IECM [49] and their lifetimes initialized based on gamma-distributed random numbers [50]. A latter attempt involves an alternative generalization of the original PSP model [46] that does not rely on conditional means, and where drift compositions remain constant during their lifetimes [51]. Recent 
versions of PSP [48,50,51] fulfill the three most important requirements in mixing models, and predict additionally a correct decay to a Gaussian distribution for chemically inert scalars. The scalar trajectories are continuous in time and multiple inert scalars evolve independently. These versions of PSP honor joint velocity-scalar statistics but compromise with respect to localness. It has been argued then that the PSP model $[48,51]$ satisfies almost all mixing models requirements.

\subsubsection{Other Mixing Models}

Several other mixing models have been proposed in the past including those based on Langevin [84] and FP equations [82,85]. The Langevin model (LM) adds a PDF dissipation term to the IEM model PDF transport equation, which leads to a decorrelation of phase space trajectories while ensuring the scalar boundedness [86]. Boundedness in this case is achieved through the use of a limiting function, which specification could be a complex task for multiscalar situations [65]. Similarly to the IEM model, LM has been also modified to account for a distribution of scalar time scales, giving rise to the so-called Extended LM (ELM) [64,65]. The FP-based models $[82,85]$ assume in turn that scalar mixing may be modeled by considering scalar diffusion in one-dimensional layer-like lamellar structures. A different stochastic mixing model is that described in [87] in which, in decaying turbulence, the variance decay rate of a scalar is modeled according to a power law. Other models [88] involve the use of scalar spectral information for the modeling of the scalar dissipation rate.

As a closure to this section, Table 1 summarizes the extent in which the main requirements associated with mixing models seem to be fulfilled by the models discussed in this work. Data shown in this table has been obtained from critical assessments of the information available in literature. The fulfillment of the requirements refers to those mixing model versions most frequently used in practice or those including the latest improvements. As it can be observed from Table 1, all mixing models reviewed present the three most fundamental characteristics required in mixing models; i.e., they satisfy mean conservation, variance decay and boundedness principles. From these three main requirements, the prediction of correct scalar variance decay rates is the most challenging as it results from the interaction of several physical phenomena. As it will observed in Section 4, different mixing models usually lead to 
different both variance decay rates and scalar PDFs. The following two requirements, Gaussianity and localness characteristics, are not satisfied by classical models such as IEM and MCD. More recent models developed for multi scalar applications include Gaussianity features in a weak (EMST and PSP) and strong (MMC and SPMM) senses. Localness in composition space has been the basis for the development of some of the latest mixing models, including EMST, MMC and SPMM, so these models satisfy this requirement. The remaining two requirements, involving dependences on scalar length scales and Reynolds (Re), Schmidt (Sc) and Damköhler (Da) numbers, have been found to be more difficult to satisfy by mixing models. Indeed IEM and MCD do not explicitly treat these requirements, although in these models scalar length scales dependences could be accounted for by using spectral models, for instance. Advanced mixing models, such as EMST, MMC, SPMM and PSP, attempt to fulfill at least partially these last two requirements. Nevertheless the extent in which these requirements are satisfied by these models need to be further verified by carrying out simulations involving a broader set of flow conditions than those already performed. Other analyses of the degree of fulfillment by mixing models of their associated requirements can be found in [48].

Table 1. Mixing models and degree of fulfillment of their associated requirements. Symbols $\mathbf{m}, \mathbf{D}$ and $\square$ refer to, respectively, requirements that are completely, partly and not fulfilled by mixing models.

\begin{tabular}{lccccccc}
\hline Requirement/Mixing model & IEM & MCD & MC & EMST & MMC & SPMM & PSP \\
\hline \hline Conservation of scalar means & - & - & - & - & - & - & - \\
Decay of scalar variance & - & - & - & - & - & - & - \\
Realizability (allowable region) & - & - & - & - & - & - & - \\
Gaussianity characteristics & $\square$ & $\square$ & - & - & - & - & - \\
Localness in scalar space & $\square$ & $\square$ & - & - & - & - & - \\
Scalar length scales dependence & $\square$ & $\square$ & - & - & - & - & - \\
Re, Sc and Da effects & $\square$ & $\square$ & - & - & - & - & - \\
\hline \hline
\end{tabular}

\section{Mixing Models Comparative Results}

Several comparisons of the mixing models performance have been carried out over the years. Both premixed and diffusion flames have been utilized for these purposes. 
The results obtained with the various mixing models have been validated using analytical solutions, DNS data and/or experimental results. Some of the results associated with the main comparative analyses of mixing models performed during the last decade are reviewed in this section. The main purpose of this review process is to emphasize the differences in the results found when using different mixing models. It is worth noticing that the illustrations containing mixing models comparative results are reproduced here exactly as they were published. This means that the nomenclature utilized in the original works has been kept in the figures discussed in this section. When required then the proper correspondence between the original nomenclature and that one used in this work is highlighted in figure captions.

Using an idealized partially stirred reactor (PaSR), Ren and Pope [89] investigated the performance of three mixing models, namely IEM [37,57], MCD [22] and EMST [24]. A detailed mechanism for hydrogen oxidation, involving 9 species and 19 reactions, and the in situ adaptive tabulation (ISAT) algorithm [90] were utilized in the simulations. A range of residence $\left(\tau_{\text {res }}\right)$ and mixing times $\left(\tau_{\text {mix }}\right)$ was studied. The performance of the mixing models was evaluated based on the resulting mixture fraction PDFs, extinction behaviors and scatter plots that characterize the PaSR statistical stationarity. Chemical equilibrium was considered for all particles as initial condition.

The mixture fraction PDFs obtained for the three different mixing models are quite different. In particular, the EMST model results in a relatively higher probability around stoichiometry as the mixture fraction variance increases. The mean temperature conditional on the mixture being stoichiometric is considered as a measure of the approach to flame extinction. Accordingly, the results show that (i) except for small values of $\xi^{\prime}$ (mixture fraction root mean square), the three mixing models exhibit substantially different extinction behaviors; and (ii) the EMST model is more resistant to global extinction than the IEM and MCD models.

Scatter plots of temperature against mixture fraction, such as those shown in Fig. 1, illustrate the qualitatively different behavior of the three mixing models. Solid lines in these scatter plots correspond to chemical equilibrium. From Fig. 1a, the reaction zone in mixture fraction space lies approximately between 0.24 and 0.50. The IEM model results (Fig. 1a) indicate that particles with composition values outside the reaction zone relax to the mean composition and are drawn away from their initial condition (equilibrium line). In addition, particles in the reaction zone react back close to their 
equilibrium values due to fast reactions. Since all particles do not lie close to the equilibrium line, it follows then that IEM fails to reproduce the expected physical behavior [89]. It is observed in Fig. 1b that the MCD [22] model mixes cold fuel with cold oxidant, thus producing unphysical cold, nonreactive mixtures within the reaction zone. Once the EMST model [24] results in all compositions being close to equilibrium (Fig. 1c), it follows that EMST produces the expected physical behavior, whereas the IEM and MCD models do not [89]. The corresponding mixture fraction PDFs are also shown in Fig. 1 and they are quite different.

Fig. 1. Mixing models performance assessed through the use of partially stirred reactors [89].

Scatter plots of temperature $(T)$ against mixture fraction $(\eta)$, and the corresponding mixture fraction PDFs obtained for $\tau_{\text {res }}=2 \times 10^{-3}(\mathrm{~s})$ and $\tau_{\text {mix }} / \tau_{\text {res }}=0.35$. In this figure MC represents the MCD [22] model discussed in the present work.

The same three mixing models, i.e., IEM [37,57], MCD [22] and EMST [24], were in turbulent diffusion flames by Mitarai et al. [91], using DNS data specifically obtained for studying local flame extinction and reignition. The tests were designed to analyze the performance of mixing models in both RAS and LES contexts. The analyses were carried out comparing mean temperature profiles, scatter plots and mixture fraction PDFs. In order to better assess the mixing models performance, the mixing frequency exact value was directly taken from the DNS results. The physical problem analyzed involved a single-step chemical reaction taking place in incompressible, isotropic, decaying turbulence. The numerical simulations were performed on a three-dimensional computational domain with periodic boundary conditions.

In RAS contexts, as illustrated in Fig. 2, the EMST mixing model reasonably accurately predicts the mean reduced temperature, whereas the IEM and MCD models tend to significantly underestimate it. It is speculated [91] that this underestimation may be related to the conserved scalar mixing frequency utilized, i.e., for reactive scalars. These mixing models do not distinguish between the mixing of passive and reactive scalars, but use identical mixing frequencies in both cases. Fig. 2 also includes CMC [75] results for comparison purposes. The overestimation of mean temperatures in this case is attributed to the fact that the $\mathrm{CMC}$ neglects the large temperature fluctuations observed during simulations. 
Fig. 2. Mean reduced temperature characterizing single step, nonpremixed reactions developing in incompressible, isotropic, decaying turbulence [91]. Left and right sides correspond to, respectively, mean (volume averaged) temperature and average temperature near the stoichiometric surface. IEM (dark circles), MCD (empty circles), EMST (asterisks). CMC [75] results (dotted line), fast chemistry limit (dashed line), DNS data (solid line).

Despite the reasonably accurate predictions of mean quantities and likewise IEM and MCD, EMST fails to reproduce the scatter plot of temperature as a function of mixture fraction. Additionally, the appearance and disappearance of cold particles, which characterizes local flame extinction and reignition, is not realistically described by any of the mixing models tested [91]. In general, the EMST mixing model yields better results than the IEM and MCD, given the mixing frequency exact values, in both RAS and LES contexts. It is believed [91] that the relative good predictions reported in literature using simple models may come from the adjustment of the mixing frequency.

Merci and co-workers performed a comparative study of the performance of mixing models in both bluff body ('Sydney Flames HM1-3') [92] and piloted jet ('Delft Flame III') [93] diffusion flames. Bluff body studies involved the Sydney flames HM1 to HM3 [94,95], which include increasingly different levels of turbulence-chemistry interaction. MCD [22] and EMST [24] were the mixing models chosen for these studies, whose performance was evaluated using scatter plots and conditional profiles (conditioned on mixture fraction). Surrounded by a co-flow air stream, the central fuel jet simulated features a mixture of hydrogen and methane (50-50\% by volume). Two different values of the empirical proportionality constant $C_{\phi}$ (1.5 and 2.0), dealing with the scalar-tomechanical time scale ratio - Eq. (26), have been studied. It has been recognized that predictions are sensitive to the choice of $C_{\phi}$, so ad hoc choices of its value may be required [96]. This is one of the reasons why sometimes different values of this model parameter are analyzed, when comparing the performance of mixing models. In order to isolate the influence of $C_{\phi}$, mixing frequency exact values are also used when possible [91].

From the series of flames considered, HM3 features the strongest turbulencechemistry interaction. However no stationary solution was obtained for this case with the use of the MCD model [22]. With EMST [24], a statistically stationary flame was obtained, but it was not possible to compare the results obtained with both mixing 
models. Results obtained from the simulations of flame HM2 illustrate that the mean mixture fraction and rms (root mean square) value of the mixture fraction fluctuations reasonably agree with the corresponding experimental data. For this particular flame, Fig. 3 shows the mean and fluctuation of temperature conditioned on mixture fraction. At the first axial position analyzed, the agreement with experimental data of the conditional mean temperature is good for all models. This agreement is better than for HM1, where the conditional temperature fluctuations obtained from experiments are much higher (presence of a slight flame lift-off). Further downstream, in terms of conditional temperatures, MCD agreement is still good, whereas an overprediction is observed around stoichiometry with the EMST model. This is due to the underprediction of the amount of local extinction associated with the EMST localness principle, made evident from the underestimation of the temperature fluctuations. Notice in Fig. 3 that there is a clear increase in conditional fluctuations around stoichiometry when the MCD model is utilized. This higher level of conditional fluctuations associated with MCD is because of its intrinsic property of random selection of particle pairs for mixing [92]. Shortly the results shown in Fig. 3 highlight the fact that differences are observed when different mixing models are utilized, especially when higher order statistics are accounted for.

Fig. 3. Results corresponding to bluff body studies, involving the Sydney flame HM2, performed to evaluate the performance of mixing models [92]. Mean (left) and rms values (fluctuation) of temperature $(T)$ conditioned on mixture fraction $(\xi)$. CD in this figure translates to MCD [22] model in this work.

When dealing with piloted jet diffusion flames ('Delft Flame III'), in addition to the MCD [22] and EMST [24] mixing models, Merci et al. [93] studied the IEM [37,57] model. These models were applied to a nonpremixed turbulent diffusion flame, featuring a strong turbulence-chemistry interaction including local extinction and reignition, known as Delft Flame III [97,98]. Particularly, the influence on the results obtained of the mixing model parameter $C_{\phi}$, Eq. (26), and the representation of pilot flames was analyzed. The analyses focused initially on the influence of $C_{\phi}$ and the pilot flame thermal power on prediction of flame attachment. The results of this preliminary analysis showed that, when the experimental pilot flame power is applied, the IEM 
leads to global flame extinction, the MCD to flame lift-off $\left(C_{\phi}=2\right)$ and the EMST to the qualitatively correct attached flame. Artificial increases in the pilot flame thermal power did not solve the IEM global extinction problem, neither led to an attached MCD-based flame. IEM was thus considered inadequate for the test case studied. The MCD flame attachment problem was corrected with a higher $C_{\phi}(=3)$ value. EMST was hardly affected by any of these modeling operations.

Results involving radial profiles of rms values of mixture fraction fluctuations show that, in general, MCD and EMST lead to an overprediction of the mixture fraction variance. An increase in $C_{\phi}$ from 2 to 3 results in a decrease of mixture fraction rms values and better agreement with experimental data. This leads however to a slight deterioration of the EMST mean temperature profiles. These EMST results show that choosing a $C_{\phi}$ value on the basis of best agreement for the mixture fraction fluctuations does not imply a similar agreement in terms of mean temperature profiles. Further analyses of the results obtained indicate that the mixture fraction variance overprediction is due to an overprediction of the turbulent diffusion transport (in physical space), which was modeled based on the gradient diffusion hypothesis. Furthermore, based on characteristic time scales, it was identified that there is much more mixing of particles from outside into a computational cell or vice versa (due to macroscopic inhomogeneity), than there is mixing within a cell (due to inhomogeneity on the molecular level). This explains the overestimated variance of mixture fraction observed. Mixture fraction rms values are influenced by the combination of production (turbulent diffusion transport) and dissipation (micromixing) processes. It is then concluded by Merci et al. [93] that it is not necessarily a good option to force agreement for mixture fraction variance by tuning the dissipation, because this may lead to unwanted enhanced local mixing and thus to poor local extinction predictions.

Local extinction was analyzed in [93] by considering scatterplots of temperature and species mass fractions versus mixture fraction, as shown in Fig. 4. From this figure it is evident that the variance of these parameters (temperature and species mass fractions), conditioned on mixture fraction (scatter in vertical direction), obtained using EMST is too low when compared to the experimental and MCD results. This EMST feature is attributed to its characteristics of localness in composition space. For the results shown in Fig. 4, typical mixture fraction rms values are equal to 0.15, which implies that the particle ensembles from each computational cell cover a significant 
width (in mixture fraction direction) in the scatterplot. In this case the differences between the EMST and MCD mixing models are therefore relevant. Because of its localness characteristics, the EMST model shows some scatter around the zone with the highest temperatures only. On the other hand, the MCD nonlocal mixing rule allows that this model has more chance to move particle compositions away from the burning region than the EMST. This is reflected in the large scatter of results observed in this case. In particular, when $C_{\phi}=2$, a strong decrease of the particle temperatures is seen to lead to local flame extinction and flame lift-off. These results seem to indicate that the failure of the MCD model with $C_{\phi}=2$ to predict an attached flame can be linked to the overprediction of the mixture fraction variance. As highlighted above, this MCD issue was solved when a higher value of this model parameter $\left(C_{\phi}=3\right)$ was utilized.

The Barlow and Frank piloted methane/air jet flames D, E and F [99,100] were utilized by Cao et al. [101] in order to investigate the performance of three mixing models, namely IEM [37,57], MCD [22] and EMST [24], and their dependence on the specified value of the mixing model parameter $C_{\phi}$, Eq. (26). In order to minimize the uncertainty in the accuracy of the chemical mechanism, the detailed GRI3.0 mechanism [102] was utilized in the simulations. Although calculations were performed for all three flames, only results for flame E, which has a significant amount of local extinction, were presented and discussed in [101]. The $C_{\phi}$ values analyzed ranged from 1.5 to 4.0. The statistics examined include conditional and unconditional mean and rms values, scatter plots and burning indexes.

Fig. 4. Mixing models comparative results obtained from piloted jet diffusion flames ('Delft Flame

III') [93]. Scatterplots of temperature (T) and water mass fraction $\left(\mathrm{Y}\left(\mathrm{H}_{2} \mathrm{O}\right)\right)$ at a given axial position $(\mathrm{x}=100 \mathrm{~mm}$ ) (experimental pilot flame power). $C D$ in this figure means MCD [22] mixing model.

For all $C_{\phi}$ values studied, flame E calculations using the EMST model yielded 'burning solutions', i.e., flames in which essentially all the fuel is converted to products far downstream the fuel injection point. In contrast, when IEM and MCD are considered, burning solutions are obtained for $C_{\phi}$ values greater than or equal to 3.3 and 3.8, but global extinction occurs for $C_{\phi}$ values less than 3.0 and 3.5, respectively. In the results shown in Fig. 5, the standard value $C_{\phi}=1.5$ is used with EMST, whereas with 
IEM and MCD the smallest values yielding burning solutions are utilized, namely $C_{\phi}=3.3$ and 3.8, respectively. Specifically Fig. 5 illustrates a comparison of the measured and computed burning indexes (BI) based on temperature and species mass fractions. In this context, BI are considered as the ratio of the conditional mean of a given parameter, in a mixture fraction interval around stoichiometry, to the corresponding value in a mildly strained nonpremixed laminar flame. Therefore, for product species, BI equal to 0 and 1 correspond to no burning and complete combustion, respectively [101].

It can be seen from in Fig. 5 that all three mixing models generally result in a reasonable agreement with the measured temperature $(\mathrm{T})$ and carbon monoxide $(\mathrm{CO})$ BI, i.e., $\mathrm{BI}(\mathrm{T})$ and $\mathrm{BI}(\mathrm{CO})$. Nevertheless, the hydroxyl $\mathrm{BI}, \mathrm{BI}(\mathrm{OH})$, computed using IEM and MCD, are significantly higher than those obtained from EMST and measurements. Notice that the $C_{\phi}$ values used for producing Fig. 5 results are those which yielded the closest agreement with the measured BI. The mixing model parameter $C_{\phi}$ affects both the BI values and the level of scalar fluctuations. For these $C_{\phi}$ values, the mean and rms mixture fraction values computed using EMST are in good agreement with experimental data. However the IEM and MCD mixing models significantly underpredict the rms mixture fraction at all locations. Based on the computed BI, and even though this is achieved with different $C_{\phi}$ values, Cao et al. [101] concluded that all three mixing models are capable of yielding levels of local extinction comparable to the experimental observations. This outcome is in line with what is highlighted in [91], where it is believed that the relative good predictions obtained using simple models may be associated with the adjustment of mixing frequency-related parameters.

Fig. 5. Axial variation of $\mathrm{BI}$ in Barlow and Frank flame $\mathrm{E}$ obtained using IEM, MCD and EMST with different $C_{\phi}$ values [101]. Solid circles are measurements and lines are joint PDF calculations. IEM with $C_{\phi}=3.3$ : dashed line with triangle, MCD with $C_{\phi}=3.8$ : dotted line with square, EMST with $C_{\phi}=1.5$ : solid line with plus.

Four different mixing models were assessed by Orbegoso and Figueira da Silva [86] using a PaSR-based approach. Models considered involve the IEM [37,57], EIEM [64-66], LM [84] and ELM [64,65]. Particularly, the degree of mixing and reaction and 
their influence on the thermochemical properties characterizing combustion processes were investigated. Results were analyzed through comparisons of both the evolution and the first and second statistical moments of the PDFs associated with each of the mixing models studied. For reference purposes, these comparisons included the use of DNS data [103] and semi-analytic steady-state solutions of the PDF corresponding to the IEM model [67]. The evolution of an inert scalar subject to homogeneous turbulent fluctuations was initially addressed. It was observed that during the early stages of mixing the scalar PDFs computed agreed well with DNS results. For large mixing times, only the LM yielded self-similar scalar PDFs consistent with a Gaussian shape. In accordance with previous results [65], EIEM and ELM, containing a description of the turbulent frequency, led in this case to a divergent, super-Gaussian behavior.

Using a PaSR the qualitative evolution of the PDF corresponding to the IEM and the stochastic mixing models was examined for situations characterized by both (i) fast mixing and reaction, and (ii) slow mixing and reaction. It was noticed that, in the former case, the shape of the steady-state PDF is only slightly modified by the choice of the mixing model. For slow reaction and mixing regimes, differences between the computed PDF shapes are found to occur, mostly at the vicinity of the unburned gases. The extended models, which contain a turbulent frequency log-normal description, are found to enhance mixing, whereas Langevin models promote reaction via an increase of scalar dissipation. A parametric analysis of the choice of the mixing model and its influence on the mean and standard deviation $(\sigma)$ of the reaction progress variable (c), which characterize the combustion processes modeled, was also carried out. For this analysis, two different values of the ratio between the mean residence time and the characteristic turbulence time, $Z=0.5$ and 2 , were studied. These $Z$ values correspond to slow and fast mixing processes, respectively. This follows from the fact that, according the modelling approach employed in [86], $Z$ is directly related to the logarithm of the turbulent frequency $(X)$. A range of residence to chemical time scale ratios was also covered, Y $[0.03,1]$, ranging from mild to intensive reaction regimes. Thus Fig. 6 shows particular results obtained from the EIM and LM simulations as part of the parametric study performed.

From Fig. 6 it is possible to see that for the case of fast mixing $(Z=2)$, the c mean values are nearly identical for the IEM model. The same occurs for the LM when the value of the parameter that controls the PDF relaxation rate in this model (do) is equal 
to 0.4 . For $\mathrm{do}=1$, larger $\mathrm{c}$ mean values characterize the $\mathrm{LM}$ in mild combustion regimes $(\mathrm{Y}<0.2)$. A different picture results for the slow mixing case shown in Fig. 6b. The reaction progress variable mean and standard deviation values, computed with IEM and $\mathrm{LM}(\mathrm{do}=0.4)$, are practically identical in intensive combustion regimes. Nevertheless, as the chemical reaction process slows, the choice of the mixing model substantially influences the $\langle c\rangle$ and $\sigma$ values. The results obtained with the use of EIEM and ELM exhibit a still larger sensitivity to the choice of both the mixing model and the LM parameter do. It is concluded that in the intense combustion regimes, i.e., when the residence to chemical time ratio is larger than 0.3 , all the studied mixing models lead to equivalent results. In the mild combustion regimes however, large differences are found to occur. In general, Langevin models (LM and ELM) lead to larger average values of the reactive scalar mean $(\langle c\rangle)$ within the PaSR. The results obtained from these models also show a dependence on the parameter that controls the reactive scalar dissipation (do). In particular, an increment of this parameter leads to an increment of the c mean values. This behavior is consistent with the increment of the averaged reaction rate with the scalar dissipation rate, which corresponds to the classic modeling of premixed turbulent combustion.

Fig. 6. Reaction progress variable mean $(\langle c\rangle)$ and standard deviation $(\sigma)$ in a PaSR operation using IEM and LM for (a) high and (b) low mixing frequencies (do is a Langevin model parameter) [86].

Meyer and Jenny [25] assessed the accuracy and computational efficiency of the PSP mixing model [46] and compared the results obtained with the corresponding ones associated with the CD [68], MCD [22], IEM [37,57] and EMST [24] models. The selected test cases involved a three-stream problem [73] and a multi-scalar test case with mean scalar gradients [24]. In the three-stream problem, involving the mixing of two inert scalars in a stationary homogeneous turbulent flow, DNS reference data [104] was utilized for reference purposes. The main results for this case show that all mixing models studied, except PSP, which is in closest agreement with DNS data, predict a constant decay rate of the rms scalar values. For the EMST model this is so by construction. The IEM is calibrated to reproduce the correct constant decay rate with a scalar energy spectrum in equilibrium [104]; and the CD-based models yield consistent 
decay rates when adequate mechanical-to-scalar timescale ratios are selected. The results also show that, after attaining a self-similar PDF shape, the PSP model predicts a DNS consistent scalar decay rate as well.

When the evolution of the joint scalar PDFs obtained using the different mixing models is analyzed, it is observed that the PSP model yields results that are in good agreement with DNS data. A similar behavior is seen from the conceptually simple Curl models. The IEM predictions are not satisfactory due to its PDF shape preserving property. The same occurs in the case of the EMST model due to the so-called stranding, i.e., unphysical formation of tree-like particle structures in scalar space. Stranding issues may be alleviated by adjusting the parameters characterizing the mixing intermittency process utilized in EMST. However this is feasible only within a limited range of values, as it may lead to reductions of the number of particles in the mixing sub-ensemble and, thus, in the localness characteristics of the model [25]. Notice that, in an inhomogeneous mixing scenario, the stranding problem is reduced since the particle transport in physical space counteracts it.

In the multi-scalar case with mean scalar gradients evaluated, a statistically stationary homogeneous turbulent flow field was also considered and constant mean scalar gradients in spatial directions were prescribed. In particular, the influence of mixing models and the number of scalars and particles on the scalar statistics and computational cost was investigated. The normalized scalar variance and kurtosis characterizing this particular case, plotted as functions of the number of scalars, are shown in Fig. 7. Manifested through the dependence on the number of scalars, this figure shows that the independence and linearity properties are not fulfilled by the EMST and early variants of the PSP mixing model. Although independence and linearity principles have been initially pointed out as requirements for mixing models, it has been shown [105] that in scalar PDF approaches mixing models need not comply with the linearity and independence hypothesis, since these are relaxed when conditional expected values are utilized. The EMST and PSP independence and linearity features could be then considered only a mild drawback for these models. It is also seen that, for a relatively large number of scalars, the EMST results approach the IEM ones, whereas the PSP variance predictions tend to the equilibrium variance obtained from DNS data [106]. Notice as well that the IEM model predicts a kurtosis of 3, which corresponds to a Gaussian distribution. Regarding the number of particles and 
computational cost, the results show that the scalar statistics have a weak dependence on the number of particles only. In addition, the CD, MCD and IEM models are computationally efficient, as their costs scale linearly with the number of scalars and particles. The PSP and EMST mixing models present higher costs however. A linear dependence on the number of particles is found for the PSP model while the EMST model leads to a nearly quadratic dependence. Moreover, for a relatively large number of scalars (> 30), both PSP and EMST present computational costs approximately proportional to the number of scalars powered to 1.5 .

Fig. 7. Non dimensional scalar variance (left) and kurtosis (right), as a function of the number of scalars $\left(n_{s}\right)$, obtained from a multi-scalar test case with mean scalar gradients [25]. IEM: (o), CD: (dash dot line), MCD: (*), EMST: (solid line), PSP: (dashed and dotted lines).

In a recent effort, Rowinski and Pope [55] carried out PDF calculations of a threestream turbulent jet in both RAS and LES contexts. The RAS-based computations involved the evaluation and comparison of three classic mixing models, namely IEM [37,57], MCD [22] and EMST [24]. One of the specific goals of this work involved the evaluation of the mixing models efficiency through comparisons of the calculated conditional diffusion with the corresponding results obtained experimentally. The non reacting flow configuration studied corresponds to the experiment conducted in [107], which consists of three concentric streams: a jet, an annulus and a coflow. It is recognized that the numerical accuracy of the calculations is affected by the (i) grid size, (ii) the number of particles per cell, (iii) the duration over which time-averaging is performed and (iv) the time step. A preliminary analysis involved then systematic tests of these parameters, such as to determine the most adequate values for the simulations. Particularly it was found that the largest source of error is in the grid resolution.

Comparative results relating the mixing models analyzed, obtained from the RAS/PDF computations carried out, are illustrated in Fig. 8. In this figure, the time averaged mass weighted mean $\left(\left\langle\tilde{\xi}_{1}\right\rangle,\left\langle\tilde{\xi}_{2}\right\rangle\right)$ and $\mathrm{rms}\left(\left\langle\widetilde{\xi}_{1}^{\prime \prime}\right\rangle,\left\langle\widetilde{\xi_{2}^{\prime \prime}}\right\rangle\right)$ mixture fractions correspond to, respectively, the jet and annulus streams. It is observed that there is a small sensitivity of the IEM-based mean mixture fractions to the $C_{\phi}$ values. In contrast to these results, Fig. 8 shows a much larger sensitivity of the rms with respect to $C_{\phi}$. Specifically, larger $C_{\phi}$ values yield a decreased rms which is expected since this model 
parameter directly impacts the scalar variance. The best agreement of the computed results with the experimental data, in terms of means and rms statistics, is obtained with a value of $C_{\phi}=1.5$. The MCD and EMST results are very similar to those obtained using the IEM mixing model.

Fig. 8. Radial profiles of time averaged mass weighted mean (a) and rms (b) mixture fractions characterizing a three-stream turbulent jet [107]. IEM, $C_{\phi}=1.5$ : solid dark line; MCD, $C_{\phi}=1.5$ : dashed dark line; EMST, $C_{\phi}=1.5$ : solid gray line; IEM, $C_{\phi}=0.5$ : dashed light gray line; IEM, $C_{\phi}=4.5$ : light gray solid line; experimental data: circles.

Even though similar results are obtained in terms of mean and rms statistics, Fig. 9 shows that there is a considerable difference in the higher order statistics associated with the different mixing models studied. The joint PDF and conditional diffusion results shown in this figure correspond to a flow location where there is significant mixing of all three streams. Regarding IEM, it is observed in Fig. 9 that this model yields a joint PDF that is more compact in composition space than the corresponding experimental one. Moreover, in contrast to the unimodal one observed experimentally, IEM results indicate a bimodal PDF. This implies that although the first two statistical moments are calculated reasonably accurately the same does not occur for higher order statistics. Additionally, in accordance with its formulation, IEM conditional diffusion values are linearly proportional to the distance from the mean value. Qualitatively the MCD model results in a joint PDF which is the most similar to what is obtained experimentally. The EMST model yields in turn a joint PDF which is much more compact than those associated with the experiments and the other mixing models analyzed.

Fig. 9. Three-stream turbulent jet - Joint PDF of mixture fractions (top) and magnitude of conditional diffusion (bottom) [107]. Circle and plus sign are mean values from experiments and

PDF-based computations, respectively. In top plots, solid lines are iso contours enclosing regions with probability 0.5 and 0.9 . In lower plots, lines with arrows are streamlines everywhere parallel to the conditional diffusion vector.

Further analyses show that the location in composition space where the dense EMST PDFs occur corresponds to the slow manifold region identified experimentally 
[107]. In this context a slow manifold is understood to be the single low dimensional manifold to which all fast manifolds, i.e., initial trajectories in mixture fraction space, are drawn to [55]. The EMST joint PDF and conditional diffusion results also show a lack of the fast manifolds observed in the experiments. It is believed thus that other physical processes not accounted for in EMST, such as differential diffusion, may be the responsible for these manifolds [55]. This study of mixing models demonstrates the strengths and weaknesses of each model, and highlights the fact that there is room for improvements in the predictions performed with these models. The simulations of the three-stream flow configuration carried out in the LES context yielded mixture fraction joint PDFs in good agreement with experimental data, better than that obtained in the RAS context with the three mixing models studied.

Other parametric studies involving mixing models constitute those presented by Meyer and Deb [50] and Meyer and Jenny [51]. In these works it is emphasized that, in joint velocity-composition PDF contexts, velocity conditioning is essential for accurate predictions of scalar statistics. Ignoring this fact in mixing models leads to significant errors in scalar statistics, including scalar mean and variances. In particular, it is shown that when velocity conditioning is neglected there are no unique $C_{\phi}$ values reproducing scalar variance data accurately. A recent effort involving comparative analyzes of the performance of mixing models is the work by Krisman et al. [58]. This study involving the IEM, MCD and EMST mixing models utilizes DNS data to provide both initial conditions and inputs needed over the course of the simulations, including mean flow velocities, mixing frequencies, and turbulent diffusion coefficients. The focus of the study is on mixing models, so attempts are made in order to both eliminate possible sources of errors (related to, for example, chemical kinetic mechanisms, turbulence modeling and numerical algorithms) and allow comparing as fair as possible the mixing models. The configuration studied corresponds to a one-dimensional, non premixed, turbulent jet flame burning either syngas or ethylene fuel streams. Parametric variations of Re and Da are also studied. For the syngas cases, the results show that, for relatively low and intermediate Re, flame extinction and reignition are successfully predicted using all three mixing models. Nevertheless all models under-predict reignition for the relatively high Re. In the ethylene fuelled cases, only the EMST model correctly predicts reignition events for the higher $\mathrm{Da}$ cases analyzed. In the lowest $\mathrm{Da}$ case studied however, the EMST over-predicts reignition and both IEM and MCD models 
under-predict it. From a parametric study performed of the mechanical-to-scalar time scale ratio $C_{\phi}$, it is concluded that this parameter is mixing model and case dependent. In the ethylene case considered then, reignition could be achieved with the IEM and MCD models by adjusting the $C_{\phi}$ values.

Similar to the studies reviewed in this section, several others have been carried out in the past in order to assess the performance of mixing models [58,108,109]. For the sake of brevity however, these studies are not detailed here. For further references regarding comparisons of mixing models performance, the interested reader is referred to Table 2 highlighting the main past studies carried out on this matter. Indeed, the purpose of this section was to emphasize the differences in the results obtained with different mixing models, rather than to discuss all parametric analyses involving these models performed so far. From the results shown in this section it may be concluded that there are significant differences in the results obtained when different mixing models are utilized. This is particularly true when higher order scalar statistics are accounted for. It has been also illustrated how the model parameters, in particular the mechanical-to-scalar time scale ratio $C_{\phi}$, influence the mixing results. A considerable effort has been undertaken in the past to determine a range of acceptable values for these model parameters without much success. In summary, work still needs to be carried out in order to develop a mixing model satisfying all desirable characteristics expected from these models. In the following section some avenues that can be explored in order to achieve this goal are discussed.

Table 2. Previous studies including comparisons of mixing models performance.

\begin{tabular}{|c|c|c|c|}
\hline Reference & Configuration & Mixing models & Main findings/conclusions \\
\hline [89] & $\begin{array}{l}\text { Partially stirred } \\
\text { reactors }\end{array}$ & $\begin{array}{l}\text { IEM, MCD, } \\
\text { EMST }\end{array}$ & $\begin{array}{c}\text { Mixing models exhibit significant differences in } \\
\text { both mixture fraction PDFs and extinction } \\
\text { behaviors }\end{array}$ \\
\hline [91] & $\begin{array}{c}\text { Turbulent } \\
\text { diffusion flames }\end{array}$ & $\begin{array}{l}\text { IEM, MCD, } \\
\text { EMST }\end{array}$ & $\begin{array}{l}\text { No mixing model realistically describes flame } \\
\text { extinction and reignition } \\
\text { EMST mixing model performs better than IEM and } \\
\text { MCD models }\end{array}$ \\
\hline$[92]$ & $\begin{array}{l}\text { Non premixed } \\
\text { bluff body flames }\end{array}$ & MCD, EMST & $\begin{array}{l}\text { Results are influenced by mixing model choice as } \\
\text { local flame extinction increases }\end{array}$ \\
\hline
\end{tabular}


[93]

Piloted jet diffusion flames

[101] Piloted jet flames

IEM, MCD, EMST

[86]

$$
\begin{aligned}
& \text { Partially stirred } \\
& \text { reactors }
\end{aligned}
$$

Three-stream problem, Mean scalar gradient configuration

$$
\begin{aligned}
& \text { Non premixed } \\
& \text { counterflow }
\end{aligned}
$$

IEM, MCD

IEM, CD, MCD, EMST, PSP

IEM, MCD, EMST

IEM, IECM, PSP

IECM, MCD, PSP configuration flame

$\begin{array}{cc}\begin{array}{c}\text { Non premixed } \\ \text { turbulent jet flame }\end{array} & \text { IEM, MCD, } \\ \text { EMST }\end{array}$

IEM model considered inadequate No scalar-to-mechanical time scale ratio value led to both mixture fraction rms and local extinction good predictions

Mixing models capable of reproducing experimental levels of local extinction Different scalar-to-mechanical time scale ratios required

Fast mixing and intense reaction: similar mixing models results

Slow mixing and mild reaction: large differences are observed

IEM, CD and MCD models are computationally efficient PSP and EMST present higher costs

Similar mixing models results are obtained in terms of mean and rms statistics

Large differences in higher order statistics are observed

PSP performs better than IECM at comparable cost Velocity conditioning is essential in joint velocitycomposition PDF contexts

Velocity-conditional MCD and PSP mixing models provide more accurate joint scalar PDF predictions (at lower computational cost) than the IECM model

Shortcomings in mixing models found Potential source: representation of scalar mixing frequency (constant ratio of scalar to flow time)

All three models give relatively similar results EMST lift-off heights shorter than the IEM and MCD ones

Scalar-to-mechanical time scale ratio optimal values are mixing model and case dependent 


\section{Prospects for Mixing Models}

It has been anticipated [2] that PDF-based methods will be adopted more broadly during the 21 st century in order to address key combustion-related issues, such as those involving the complex interactions among turbulence, gas-phase chemistry, liquid fuel sprays, soot and thermal radiation. In the particular formulation used for turbulent combustion modeling known as transported PDF method, the mixing models dealing with the PDF transport due to the molecular diffusivity remain the weakest link [4]. Currently there is no mixing model presenting all desirable characteristics expected from these models. This implies that the development of mixing models is an open area for research. This is particularly true in the case of the relative new approaches being utilized, involving Eulerian Monte Carlo [6-8] and sparse-Lagrangian Monte Carlo [74,76] methods.

In general mixing models should rely on sound physical grounds. This allows both performing a straightforward validation of their results by using experimental or DNS data, and obtaining an overall picture of the molecular diffusion processes that they are supposed to mimic. One route that could be further explored is that in which the effects of the turbulent frequency scale distribution on scalar mixing are included [64-66]. This approach is based on the fact that, although molecular mixing is a small scale phenomenon, the local scalar dissipation frequencies determining roughly its intensity, depend on the scalar structures influenced by the full spectra of turbulence. It has been thus proposed to replace the single frequency scale used in most mixing models by a stochastic distribution of this parameter. The challenge here is how to properly model this stochastic process. It has been emphasized [4] as well that the lack of information regarding the joint scalar dissipation rates in most mixing models may lead to unphysical predictions. This means that mixing models should contain information about small scale correlations between scalar gradients. Improving upon existing mixing models implies then finding accurate models for the scalar dissipation rates and their dependence on the chemical source term [4]. Notice as well that, as discussed in [110], the problem raised by the numerical prediction of the mean chemical reaction rate has led to the development of several modeling approaches for the scalar dissipation rates. The works dealing with these modeling approaches emphasize the existing relationship 
between mean chemical reaction and scalar dissipation rates. The outcomes from these works could also contribute thus for the development of improved mixing models.

The mixture fraction length scale distribution often appears in the form of the fluctuating scalar dissipation rate in turbulent combustion models [4]. Because of the importance of accurately modeling the scalar dissipation rates, the scalar length scale distribution is also thus a key aspect to be considered in mixing models. It has been shown indeed that accounting for the evolution of the scalar length scale distribution is crucial for predicting the correct decay rate of scalar variance [111]. The scalar length scales can be linked to the experimental results obtained recently by Cai et al. [107], from the characterization of three-scalar mixing processes in a turbulent coaxial jet configuration. In this experimental work, it has been found that (i) the diffusion velocity streamlines in scalar space generally quickly converge to a manifold along which they continue at a lower rate. Additionally, (ii) the mixing processes during the approach to the manifold cannot be modelled by using different dissipation time scales alone. Because of the particular three-stream flow configuration analyzed, mixing between two of the scalars can occur only through the third one, i.e., through a preferential mixing path in scalar space. This mixing path is indeed challenging for mixing models, since most of them use only scalar-space variables and disregard the spatial (physical-space) scalar structure.

One of the mixing models considering, directly or indirectly, both scalar and spatial spaces is the MMC model $[73,74,76]$. In this particular model, in order to preserve localness, only particles that are close in both reference and physical spaces are allowed to be mixed. Thus this model accounts for the spatial scalar structure mentioned above. It is worth highlighting that, in order to provide for locality characteristics to a given mixing model, it is necessary to first define the particles neighborhood. This means that, for a given particle, its corresponding neighbors need to be firstly identified. The definition of a particle neighborhood can be done directly in scalar space (EMST [24]), reference variables space, and/or physical space (MMC [73,74,76]). For single scalar cases this neighborhood definition can be reduced to a simple particle ordering according to the values of given parameter. For multi scalar situations however, this process can be complex and expensive in computational terms. Devising a relative simple scalar mixing model which ensures localness remains thus a challenge nowadays. 
The use of a non-traditional mixing approach for simulating eventually turbulent reacting flows has been proposed recently [112]. Traditional mixing as discussed in this review work is conservative, which means that the total amount of a scalar is preserved during a mixing event. In contrast, the non-traditional mixing known as nonconservative or competitive mixing considers a more general mixing that does not possess these conservative properties. In short, in non-conservative mixing, the particle post-mixing average becomes biased towards one of the particles participating in the mixing process [112]. Competitive mixing has been used in simulations emulating the propagation of combustion waves in turbulent premixed combustion under the flamelet regime [113]. Competitive mixing is seen, in this case, as the superposition of conservative mixing followed by the rapid evolution of the particles into the burned state. The results obtained for this particular application are shown to be consistent with the corresponding experimental evidence. This mixing approach constitutes an alternative route towards the development of mixing models that could be further explored in future. Additional discussions on the concept of mixing in different contexts, including mechanical mixing, ergodic theory, turbulent combustion modeling and complex competitive systems, can be found in [114].

The remaining challenges facing turbulent combustion models have been reviewed in [115], from the point of view of the implied manifolds in the species space. These challenges are related to the presence, in the combustion processes, of small scales and many chemical species, as well as to the coupled processes of reaction and molecular diffusion. Turbulent combustion involves a large range of scales, from those of the apparatus size down to the smallest turbulent and reaction-zone scales. The efficient representation of the small scales inevitably requires the use of a statistical approach for the modelling of the small-scale processes. This implies in turn that the coupled processes of reaction and molecular diffusion have to be modeled [115]. Mixing models such as those discussed above are utilized for these purposes. Notice that relative simple models, including IEM [37,57] and MCD [22], do not account for the effects that reaction may have such as to steepen scalar gradients and, hence, to influence molecular mixing. Nevertheless more sophisticated models such as EMST [24] and MMC [73,74,76] do account for these effects [115], which gives them good prospects for further improvements. 
An important subject that has been addressed in [115] relates to the examination of the processes of reaction and molecular diffusion from the perspective of manifolds in the species space. Different types of manifolds used in turbulent combustion, including skeletal, reaction-diffusion, conditional and empirical manifolds, have been thus discussed. More specifically, it has been shown that empirical manifolds can be constructed based on composition samples obtained from experimental or DNS data. Particular results illustrate that DNS data corresponding to relatively simple flames are not well approximated by low dimensional empirical manifolds. Since flamelet-like models assume that computed compositions are confined to a low dimensional manifold, it is doubted in [115] on their applicability to simple flame configurations, and yet more so to the more complex ones. Certainly these manifold-based approaches open other avenues to be explored in order to improve mixing models currently used in simulations of turbulent combustion processes.

Thinking out of the box may also help to further improve current mixing models. In this sense the study of coherent structures, which are distinguishable regions of the flow field sharing common properties and correlated in space and time [116], have the potential to open exciting avenues for mixing models research. It is known that coherent structures play a key role in turbulent transport and mixing $[117,118]$. However, the main issue that remains is the determination of these coherent structures based on experimental/numerical data. In Eulerian contexts, a problem arises when defining the criteria for coherence as different definitions favor different structures. Besides, Eulerian coherence indicators are frame dependent and hence often unable to capture intrinsic flow properties in unsteady flows [117]. Lagrangian coherent structures (LCS) $[119,120]$ have evolved thus from a need to formally define intrinsic structures governing flow transport within fluid flows. This means that LCS are expected to establish frame-independent criteria allowing unambiguous definition of coherent structures based on their influence on flow transport [121]. When using LCS fluid motion is seen from a Lagrangian perspective, which means that LCS can be defined as distinguished sets of fluid particles [117]. As highlighted in literature [119], LCS have a direct impact on fluid mixing due to their stability properties. In laminar flows LCS can be determined using chaotic advection methods [122]. Nevertheless, it is more difficult to identify LCS from turbulent flow experiments, as the stability properties of individual fluid particles are difficult to establish [117]. Ultimately, it is expected that the proper 
identification and eventually prediction of LCS could help to better understand and effectively exploit fluid flows in engineering.

Following a line of reasoning similar to LCS and flow patterns, another route that can be further explored is that accounting for the interaction of combustion thermochemical processes with the local geometries of both scalar fields and flow topologies [123]. This approach has a great potential to contribute to the development of more accurate mixing models, as it is intended to include a solid grasp of the physical process that mixing models are supposed to represent. Since the pioneering work of Chong et al. [124], who studied and classified local small-scale topologies for both compressible and incompressible flows, based on the velocity-gradient tensor invariants, several other investigations have been carried out on this subject. Most of these works have been performed for constant density flows. Accordingly, the turbulent mixing of a single reacting scalar in a statistically homogeneous constant density flow is addressed in [125]. It is shown that scalar mixing depends on the topologies of the velocity field, especially when the velocity gradient invariants are large, indicating that small scale intermittency plays a role in turbulent mixing mechanisms. One recent study about local flow topologies and scalar structures [123] considers the case of a turbulent premixed flame. The main results of this work indicate that thermochemical processes tend to smooth out highly contorted iso-scalar surfaces in some flow regions. Besides particular flow topologies correlate with large values of the strain rate tensor, providing thus hints on the flow topologies fostering scalar mixing. Molecular mixing is a small length scale process, so the interaction between small scale flow topologies and scalar structures needs to be accounted for when developing mixing models. Furthermore, given the fact that chemistry can enhance existing scalar gradients, the characteristic mixing time (or frequency) can be strongly influenced by combustion. The presence of chemical reactions cannot be ignored then in mixing models. It seems that the main focus of investigations has been so far on the use of a large scale characteristic time, instead of, for example, Kolmogorov strain rates or molecular diffusion coefficients. This needs to be addressed indeed should better mixing models are to be developed in future.

As it has been pointed out throughout this section, several approaches have the potential to make contributions to the development of better and more comprehensive mixing models and some of them seem to be more promising than the others. In a more 
personal level, we believe that generality and simplicity should be the main driving forces for the development of new mixing models. We have seen that by tuning model constants it has been possible to obtain in some situations results that reasonably agree with experimental or DNS data, at least in terms of low order statistical moments. Nevertheless it seems awkward to perform a tuning process before using these models. Generality, as well as simplicity or ease of use, are then key for future developments involving mixing models. Having this in mind, as it would allow representing more accurately the physical phenomena involved, we definitely believe that more physics needs to be embedded in mixing models. In particular local length or time scales, relating small scale flow topologies and scalar structures, need to be accounted for in mixing models. Accordingly, we consider that one of the most promising avenues for the development of improved mixing models is that involving feature extraction [126,127] from large scale experimental or DNS data. Additionally, regardless of the methodology used for analyzing data, recognizing patterns and extracting relevant features from it, it is crucial to understand the local relationship between mixing and chemical reactions, i.e., the interaction of chemical processes with local geometries of scalar and flow fields. Ultimately, we expect that both the proper identification of relevant features from large scale data, and the understanding of the interaction among turbulence, mixing and reaction allow the conception and development of simple, general and more accurate mixing models than those currently available.

\section{Conclusions}

In this work a review of the main mixing models utilized for closure purposes in Lagrangian transported PDF formulations focused on turbulent reacting flows was presented. These models provide in practice a closure to the PDF transport equation open terms that represent the transport of the PDF due to molecular diffusion. Over the years several mixing models with different degrees of complexity have been developed. The review carried out included models in which the composition of a particle changes not only essentially independently of that of the other particles, but also through direct interaction with other particles. Main advantages and shortcomings of the mixing models reviewed were highlighted as required. Because of the current interest in solving PDF transport equations using Lagrangian Monte Carlo techniques in hybrid 
Euler/Lagrange approaches, a particular emphasis was put on those models applicable to particle-based schemes. In the last part of the article both comparative results of mixing models performance, and prospects for the mixing models were discussed to complement the literature review carried out.

Despite the effort that has been devoted to the development of more capable mixing models, there is no mixing model presenting all desirable characteristics. Even more, there are significant differences in the results obtained when different mixing models are utilized, especially when higher order scalar statistics are accounted for. It has been observed that particular model parameters influence the mixing results. This is particularly true in the case of the mechanical-to-scalar time scale ratio, which determines the transported scalar mixing rates. In the past, several works have been focused on the determination of acceptable values for this parameter, but the results have not been as expected. Results show indeed that the value of this particular model parameter is both mixing model and case dependent. It is therefore concluded that work still needs to be carried out in order to develop a mixing model satisfying all desirable characteristics expected from these models. Several avenues can be further explored in order to achieve this goal. These potential routes include those accounting for spatial scalar structures and both scalar length and turbulent frequency scales distributions. Other approaches based on competitive mixing, manifold-based features and Lagrangian coherent structures may also bring further improvements upon existing mixing models. From all potential approaches, those embedding more physics into mixing models seem to be the most promising, as they are supposed to account more properly for the interaction among turbulence, mixing and chemical reactions. The development of a sound mixing model will allow eventually removing one of the largest sources of modeling uncertainty in PDF-based calculations.

\section{Acknowledgments}

This work has been supported by Petrobras under the technical monitoring of Dr. Ricardo Serfaty (Project: Development of a modeling technique for turbulent combustion based on an Eulerian/Lagrangian approach - Phase II, Contract No.: 0050.0080122.12.9). During this work Luís Fernando Figueira da Silva was on leave from the Institut Pprime (Centre National de la Recherche Scientifique, France). We 
extend our gratitude and thankfulness to Dr. Vladimir A. Sabel'nikov (Office National d'Etudes et de Recherches Aérospatiales, France) for his insightful comments.

\section{References}

1. World Energy Outlook 2013, International Energy Agency <www.worldenergyoutlook.org>.

2. Haworth, D. C., Progress in probability density function methods for turbulent reacting flows, Progress in Energy and Combustion Science 36: 168-259 (2010).

3. Pope, S. B., Turbulent Flows, Cambridge University Press (2000).

4. Fox, R. O., Computational Models for Turbulent Reacting Flows, Cambridge University Press (2003).

5. Pope, S., PDF methods for turbulent reactive flows, Progress in Energy and Combustion Science 11: 119-192 (1985).

6. Valiño, L., A field Monte Carlo formulation for calculating the probability density function of a single scalar in a turbulent flow, Flow, Turbulence and Combustion 60: 157-172 (1998).

7. Sabel'nikov, V., Soulard, O., Rapidly decorrelating velocity field model as a tool for solving one-point Fokker-Planck equations for probability density functions of turbulent reactive scalars, Physical Review E 72: 016301 (2005).

8. Sabel'nikov, V., Soulard, O., Eulerian (Field) Monte Carlo methods for solving PDF transport equations in turbulent reacting flows, Handbook of Combustion 3: 75-119 (2010).

9. Jaishree, J., Lagrangian and Eulerian probability density function methods for turbulent reacting flows, Ph.D. thesis, The Pennsylvania State University, University Park, PA, USA (2011).

10. Jaishree, J., Haworth, D. C., Comparisons of Lagrangian and Eulerian PDF methods in simulations of non-premixed turbulent jet flames with moderate-tostrong turbulence-chemistry interactions, Combustion Theory and Modelling 16: 435-463 (2012). 
11. Muradoglu, M., Jenny, P., Pope, S. B., Caughey, D. A., A consistent hybrid finitevolume/particle method for the PDF equations of turbulent reactive flows, Journal of Computational Physics 154: 342-371 (1999).

12. Jenny, P., Pope, S. B., Muradoglu, M., Caughey, D. A., A hybrid algorithm for the joint PDF equation of turbulent reactive flows, Journal of Computational Physics 166: 218-252 (2001).

13. Jenny, P., Muradoglu, M., Liu, K., Pope, S. B., Caughey, D. A., PDF simulations of a bluff-body stabilized flow, Journal of Computational Physics 169: 1-23 (2001).

14. Andrade, F. O., Figueira da Silva, L. F., Mora, A., Large eddy simulation of turbulent premixed combustion at moderate Damköhler numbers stabilized in a high-speed flow, Combustion Science and Technology 183: 645-664 (2011).

15. Vedovoto, J. M., da Silveira Neto, A., Figueira da Silva, L. F., Mura, A., Influence of synthetic inlet turbulence on the prediction of low Mach number flows, Computer \& Fluids (available online) (2014).

16. Raman, V., Pitsch, H., Fox, R. O., Hybrid large-eddy simulation/lagrangian filtered-density-function approach for simulating turbulent combustion, Combustion and Flame 143: 56-78 (2005).

17. Raman, V., Fox, R. O., Harvey, A. D., Hybrid finite-volume/transported PDF simulations of a partially premixed methane-air flame, Combustion and Flame 136: 327-350 (2004).

18. Muradoglu, M., Liu, K., Pope, S. B., PDF modeling of a bluff-body stabilized turbulent flame, Combustion and Flame 132: 115-137 (2003).

19. James, S., Zhu, J., Anand, M. S., Large eddy simulations of turbulent flames using the filtered density function model, Proceedings of the Combustion Institute 31: 1737- 1745 (2007).

20. Pope, S. B., Mapping closures for turbulent mixing and reaction, Theoretical and Computational Fluid Dynamics 2: 255-270 (1991).

21. Norris, A. T., Pope, S. B., Turbulent mixing model based on ordered pairing, Combustion and Flame 83: 27-42 (1991). 
22. Janicka, J., Kolbe, W., Kollmann, W., Closure of the transport-equation for the probability density-function of turbulent scalar fields, Journal of Non-Equilibrium Thermodynamics 4: 47-66 (1979).

23. Dopazo, C., Recent developments in PDF methods, In: Libby, P. A., Williams, F.A. (Ed.), Turbulent reacting flows, Academic Press (1994).

24. Subramaniam, S., Pope, S. B., A mixing model for turbulent reactive flows based on Euclidean minimum spanning trees, Combustion and Flame; 115: 487-514 (1998).

25. Meyer, D. W., Jenny, P., Micromixing models for turbulent flows, Journal of Computational Physics 228: 1275-1293 (2009).

26. Williams, F. A., Combustion Theory, $2^{\text {nd }}$ Ed., Benjamin Cummings (1985).

27. Poinsot, T., Veynante, D., Theoretical and Numerical Combustion, $2^{\text {nd }}$ Ed., R. T. Edwards Inc. (2005).

28. Sheikhi, M. R. H., Givi, P., Pope, S. B., Frequency-velocity-scalar filtered mass density function for large eddy simulation of turbulent flows, Physics of Fluids 21: 075102 (2009).

29. Jaberi, F. A., Colucci, P. J., James, S., Givi, P., Pope, S. B., Filtered mass density function for large-eddy simulation of turbulent reacting flows, Journal of Fluid Mechanics 401: 85-121 (1999).

30. Lundgren, T. S., Distribution functions in the statistical theory of turbulence, Physics of Fluids 10: 969-975 (1967).

31. Pope, S. B., Transport equation for the joint probability density function of velocity and scalars in turbulent flow, Physics of Fluids 24: 588-596 (1981).

32. Sheikhi, M. R. H., Givi, P., Pope, S. B., Velocity-scalar filtered mass density function for large eddy simulation of turbulent reacting flows, Physics of Fluids 19: 095106 (2007).

33. Colucci, P. J., Jaberi, F. A., Givi, P., Pope, S. B., Filtered density function for large eddy simulation of turbulent reacting flows, Physics of Fluids 10: 499-515 (1998). 
34. Risken, H., The Fokker-Planck Equation, Methods of Solution and Applications, Springer (1989).

35. Gardiner, C. W., Handbook of Stochastic Methods, Springer-Verlag (1990).

36. Haworth, D. C., Pope, S. B., A generalized Langevin model for turbulent flows, Physics of Fluids 29, 387-405 (1986).

37. Villermaux, J., Devillon, J. C., Représentation de la coalescence et de la redispersion des domaines de ségrégation dans un fluide par un modèle d'interaction phénoménologique, Proceedings of the 2nd International Symposium on Chemical Reaction Engineering, New York, USA (1972).

38. McDermott, R., Pope, S. B., A particle formulation for treating differential diffusion in filtered density function methods, Journal of Computational Physics 226: 947-993 (2007).

39. Viswanathan, S., Pope, S. B., Turbulent dispersion from line sources in grid turbulence, Physics of Fluids 20: 101514 (2008).

40. Pope, S. B., A Lagrangian two-time probability density function equation for inhomogeneous turbulent flows, Physics of Fluids 26, 3448-3450 (1983).

41. Meyers, R. E., O’Brien, E. E., The joint PDF of a scalar and its gradient at a point in a turbulent fluid, Combustion Science and Technology 26: 123-134 (1981).

42. Babenko, V. A., Frost, V. A., Modeling of turbulent reacting flows on the base of the equation for the scalar field correlation function, International Journal of Heat and Mass Transfer 52: 3314-3319 (2009).

43. Zhang, Y. Z., Hybrid particle/finite-volume PDF methods for three-dimensional time-dependent flows in complex geometries, Ph.D. thesis, The Pennsylvania State University, University Park, PA, USA (2004).

44. Xu, J., Pope S. B., Assessment of numerical accuracy of pdf monte carlo methods for turbulent reacting flows, Journal of Computational Physics 152:192-230 (1999).

45. Zhang, Y. Z., Haworth, D. C., A general mass consistency algorithm for hybrid particle/finite-volume pdf methods, Journal of Computational Physics 194:156193 (2004). 
46. Meyer, D. W., Jenny, P., A mixing model for turbulent flows based on parameterized scalar profiles, Physics of Fluids 18: 035105 (2006).

47. Pope, S. B., Turbulent flows, Cambridge University Press, Cambridge, UK (2000).

48. Meyer, D. W., A new particle interaction mixing model for turbulent dispersion and turbulent reactive flows, Physics of Fluids 22:035103 (2010).

49. Pope, S. B., The vanishing effect of molecular diffusivity on turbulent dispersion: implications for turbulent mixing and the scalar flux, Journal of Fluid Mechanics 359: 299-312 (1998).

50. Meyer, D. W., Deb, R., Modeling molecular mixing in a spatially inhomogeneous turbulent flow, Physics of Fluids 24: 025103 (2012).

51. Meyer, D. W., Jenny, P., Accurate and computationally efficient mixing models for the simulation of turbulent mixing with PDF methods, Journal of Computational Physics 247: 192-207 (2013).

52. Dialameh, L., Sundaram, B., Cleary, M. J., Klimenko, A. Y., Differential diffusion of passive scalars with MMC mixing model in isotropic turbulent flow, Proceedings of the 18th Australasian Fluid Mechanics Conference, Launceston, Australia (2012).

53. Flagan, R. C., Appleton, J. P., A stochastic model of turbulent mixing with chemical reaction: Nitric oxide formation in a plug-flow burner, Combustion and Flame 23: 249-267 (1974).

54. Wang, H., Pope, S. B., Large eddy simulation/probability density function modeling of a turbulent $\mathrm{CH} 4 / \mathrm{H} 2 / \mathrm{N} 2$ jet flame, Proceedings of the Combustion Institute 33: 1319- 1330 (2011).

55. Rowinski, D., Pope, S. B., An investigation of mixing in a three-stream turbulent jet, Physics of Fluids 25: 105105 (2013).

56. Bisetti, F., Chen, J. Y., LES of Sandia flame D with Eulerian PDF and finite-rate chemistry, Fall Meeting Western States Section of the Combustion Institute, Stanford Univeristy, California, USA (2005). 
57. Dopazo, C., O'Brien, E. E., An approach to the autoignition of a turbulent mixture, Acta Astronautica 1: 1239-1266 (1974).

58. Krisman, A., Tang, J. C. K., Hawkes, E. R., Lignell, D. O., Chen, J. H., A DNS evaluation of mixing models for transported PDF modelling of turbulent nonpremixed flames, Combustion and Flame, Accepted for publication (2014).

59. Stöllinger, M., Heinz, S., Evaluation of scalar mixing and time scale models in PDF simulations of a turbulent premixed flame, Combustion and Flame 157: 1671-1685 (2010).

60. Heinz, S., Statistical mechanics of turbulent flows, Springer-Verlag (2003).

61. Fox, R. O., On velocity-conditioned scalar mixing in homogeneous turbulence, Physics of Fluids 8: 2678-2691 (1996).

62. Heinz, S., On Fokker-Planck equations for turbulent reacting flows, Part 1: Probability density function for Reynolds-averaged Navier-Stokes equations, Flow, Turbulence and Combustion 70: 115-152 (2003).

63. Heinz, S., On Fokker-Planck equations for turbulent reacting flows, Part 2: Filter density function for large eddy simulation, Flow, Turbulence and Combustion 70: 153-181 (2003).

64. Sabel'nikov, V., Gorokhovski, M., Extended LMSE and Langevin models of the scalar mixing in the turbulent flow, Second International Symposium on Turbulence and Shear Flow Phenomena, Royal Institute of Technology (KTH), Stockholm, Sweden (2001).

65. Soulard, O., Sabel'nikov, V., Gorokhovski, M., Stochastic scalar mixing models accounting for turbulent frequency multiscale fluctuations, International Journal of Heat and Fluid Flow 25: 875-883 (2004).

66. Sabel'nikov, V., Gorokhovski, M., Baricault, N., The extended IEM mixing model in the framework of the composition PDF approach: applications to diesel spray combustion, Combustion Theory and Modelling 10: 155-169 (2006).

67. Sabel'nikov, V., Figueira da Silva, L. F., Partially stirred reactor: study of the sensitivity of the Monte-Carlo simulation to the number of stochastic particles 
with the use of a semi-analytic, steady-state, solution to the pdf equation, Combustion and Flame 129: 164-178 (2002).

68. Curl, R. L., Dispersed phase mixing: I. Theory and effects in simple reactors. AIChE Journal 9: 175-181 (1963).

69. Dopazo, C., Relaxation of initial probability density functions in the turbulent convection of scalar fields, Physics of Fluids 22: 20-30 (1979).

70. Pope, S. B., An improved turbulent mixing model, Combustion Science and Technology 28: 131-145 (1982).

71. Chen, H., Chen, S., Kraichnan, R. H., Probability distribution of a stochastically advected scalar field, Physical Review Letters 63: 2657-2660 (1989).

72. Pope, S. B., A model for turbulent mixing based on shadow-position conditioning, Physics of Fluids 25, 110803 (2013).

73. Klimenko, A. Y., Pope, S. B., The modeling of turbulent reactive flows based on multiple mapping conditioning, Physics of Fluids 15: 1907-1925 (2003).

74. Cleary, M. J., Klimenko, A. Y., Janicka, J., Pfitzner, M., A sparse-Lagrangian multiple mapping conditioning model for turbulent diffusion flames, Proceedings of the Combustion Institute 32: 1499-507 (2009).

75. Klimenko, A. Y., Bilger, R. W., Conditional moment closure for turbulent combustion, Progress in Energy and Combustion Science 25: 595-687 (1999).

76. Cleary, M. J., Klimenko, A. Y., A generalised multiple mapping conditioning approach for turbulent combustion, Flow, Turbulence and Combustion 82: 477491 (2009).

77. Wandel, A. P., Klimenko, A. Y., Testing multiple mapping conditioning mixing for monte carlo probability density function simulations, Physics of Fluids 17: 128105 (2005).

78. Raman, V., Pitsch, H., “A consistent LES/filtered-density function formulation for the simulation of turbulent flames with detailed chemistry", Proceedings of the Combustion Institute 31: 1711-1719 (2007). 
79. Yang, Y., Wang, H., Pope, S. B., Chen, J. H., "Large-eddy simulation/probability density function modeling of a non-premixed $\mathrm{CO} / \mathrm{H} 2$ temporally evolving jet flame", Proceedings of the Combustion Institute 34: 1241-1249 (2013).

80. Cleary, M. J., Klimenko, A. Y., A detailed quantitative analysis of sparseLagrangian filtered density function simulations in constant and variable density reacting jet flows, Physics of Fluids 23: 115102 (2011).

81. Jayesh, Pope, S. B., Stochastic model for turbulent frequency, Technical Report No. FDA 95-05, Cornell University, USA (1995).

82. Fox, R. O., The Fokker-Planck closure for turbulent molecular mixing: passive scalars, Physics of Fluids 4: 1230-1244 (1992).

83. Meyer, D. W., Jenny, P., A mixing model providing joint statistics of scalar and scalar dissipation rate, Proceedings of the Combustion Institute 32: 1613-1620 (2009).

84. Valiño L., Dopazo, C., A binomial Langevin model for turbulent mixing. Physics of Fluids 3: 3034-3037 (1991).

85. Fox, R. O., Improved Fokker-Planck model for the joint scalar, scalar gradient PDF, Physics of Fluids 6: 334-348 (1994).

86. Orbegoso, E. M., Figueira da Silva, L. F., Study of stochastic mixing models for combustion in turbulent flows, Proceedings of the Combustion Institute 32: 15951603 (2009).

87. Fedotov, S., Ihme, M., Pitsch, H., Stochastic mixing model with power law decay of variance, Physical Review E 71: 016310 (2005).

88. Fox, R. O., The Lagrangian spectral relaxation model of the scalar dissipation in homogeneous turbulence, Physics of Fluids 9: 2364-2386 (1997).

89. Ren, Z., Pope, S. B., An investigation of the performance of turbulent mixing models, Combustion and Flame 136: 208-216 (2004).

90. Pope, S. B., Computationally efficient implementation of combustion chemistry using in situ adaptive tabulation, Combustion Theory and Modelling 1: 41-63 (1997). 
91. Mitarai, S., Riley, J. J., Kosály, G., Testing of mixing models for Monte Carlo probability density function simulations, Physics of Fluids 17: 047101 (2005).

92. Merci, B., Roekaerts, D., Naud, B., Pope, S. B., Comparative study of micromixing models in transported scalar PDF simulations of turbulent nonpremixed bluff body flames, Combustion and Flame 146: 109-130 (2006).

93. Merci, B., Roekaerts, D., Naud, B., Study of the performance of three micromixing models in transported scalar PDF simulations of a piloted jet diffusion flame (“Delft Flame III”), Combustion and Flame 144: 476-493 (2006).

94. Dally, B. B., Masri, A. R., Barlow, R. S., Fiechtner, G. J., Fletcher, D. F., Instantaneous and mean compositional structure of bluff-Body stabilized nonpremixed flames, Combustion and Flame 114: 119-148 (1998).

95. Dally, B. B., Fletcher, D. F., Masri, A. R., Flow and mixing fields of turbulent bluff-body jets and flames, Combustion Theory and Modelling 2: 193-219 (1998).

96. Pope, S. B., Mixing model performance in the calculation of nonpremixed piloted jet flames, Proceedings of the Sixth International Workshop on Measurement and Computation of Turbulent Nonpremixed Flames, Combustion Institute, Sapporo, Japan (2002).

97. Peeters, T. W. J., Stroomer, P. P. J., de Vries, J. E., Roekaerts, D., Hoogendoorn, C. J., Comparative experimental and numerical investigation of a piloted turbulent natural-gas diffusion flame, Proceedings of the Combustion Institute 25: 12411248 (1994).

98. van Veen, E. H., Roekaerts, D., On the accuracy of temperature measurements in turbulent jet diffusion flames by coherent Anti-Stokes Raman spectroscopy, Combustion Science and Technology 175: 1893-1914 (2003).

99. Barlow, R. S., Frank, J. H., Effects of turbulence on species mass fractions in methane/air jet flames, Symposium (International) on Combustion 27: 1087-1095 (1998).

100. Frank, J. H., R.S. Barlow, R. S., Lundquist, C., Radiation and nitric oxide formation in turbulent non-premixed jet flames, Proceedings of the Combustion Institute 28: 447-454 (2000). 
101. Cao, R. R., Wang, H., Pope, S. B., The effect of mixing models in PDF calculations of piloted jet flames, Proceedings of the Combustion Institute 31: 1543-1550 (2007).

102. GRI-Mech Web site, available at http://www.me.berkeley.edu/gri-mech/.

103. Eswaran, V., Pope, S. B., Direct numerical simulations of the turbulent mixing of a passive scalar, Physics of Fluids 31: 506-520 (1988).

104. Juneja, A., Pope, S. B., A DNS study of turbulent mixing of two passive scalars, Physics of Fluids 8: 2161-2184 (1996).

105. Hierro, J., Dopazo. C., Scalar probability density function mixing models need not comply with the linearity and independence hypothesis, Physics of Fluids 16: 1811 (2004).

106. Overholt, M. R., Pope, S. B., Direct numerical simulation of a passive scalar with imposed mean gradient in isotropic turbulence, Physics of Fluids 8: 3128-3148 (1996).

107. Cai, J., Dinger, M. J., Li, W., Carter, C. D., Ryan, M. D., Tong, C. Experimental study of three-scalar mixing in a turbulent coaxial jet, Journal of Fluid Mechanics 685: 495-531 (2011).

108. Blouch, J. D., Chen, J. Y., Law, C. K., A joint scalar PDF study of nonpremixed hydrogen ignition, Combustion and Flame 135: 209-225 (2003).

109. Cao, R. R., Pope, S. B., Masri, A. R., Turbulent lifted flames in a vitiated coflow investigated using joint PDF calculations, Combustion and Flame 142: 438-453 (2005).

110. Dong, H. Q., Robin, V., Mura, A., Champion, M., Analysis of algebraic closures of the mean scalar dissipation rate of the progress variable applied to stagnating turbulent flames, Flow, Turbulence and Combustion 90: 301-323 (2013).

111. Cremer, M. A., McMurtry, P. A., Kerstein, A. R., Effects of turbulence lengthscale distribution on scalar mixing in homogeneous turbulent flow, Physics of Fluids 6: 2143-2153 (1994).

112. Klimenko, A. Y., Conservative and competitive mixing and their applications, Physica Scripta T142: 014054 (2010) 
113. Klimenko, A. Y., Pope, S. B., Propagation speed of combustion and invasion waves in stochastic simulations with competitive mixing, Combustion Theory and Modeling 16: 679-714 (2012).

114. Klimenko, A. Y., What is mixing and can it be complex?, Physica Scripta T155: 014047 (2013).

115. Pope, S. B., Small scales, many species and the manifold challenges of turbulent combustion, Proceedings of the Combustion Institute 34: 1-31 (2013).

116. Ouellette, N. T., On the dynamical role of coherent structures in turbulence, Comptes Rendus Physique 13: 866-887 (2012).

117. Mathur, M., Haller, G., Peacock, T., Ruppert-Felsot, J. E., Swinney, H. L., Uncovering the Lagrangian Skeleton of Turbulence, Physical Review Letters 98: 144502 (2007).

118. Wiggins, S., Coherent structures and chaotic advection in three dimensions, Journal of Fluid Mechanics 654: 1-4 (2010).

119. Haller, G., Lagrangian coherent structures from approximate velocity data, Physics of Fluids 14: 1851-1861 (2002)

120. Speetjens, M. F. M., Lauret, M., Nijmeijer, H., Anderson, P. D., Footprints of Lagrangian flow structures in Eulerian concentration distributions in periodic mixing flows, Physica D: Nonlinear Phenomena 250: 20-33 (2013).

121. Peacock, T., Dabiri, J., Introduction to Focus Issue: Lagrangian Coherent Structures, Chaos: An Interdisciplinary Journal of Nonlinear Science 20: 017501 (2010).

122. Pouransari, Z., Speetjens, M. F. M., Clercx, H. J. H., Formation of coherent structures by fluid inertia in three-dimensional laminar flows, Journal of Fluid Mechanics 654: 5-34 (2010).

123. Cifuentes, L., Dopazo. C., Martin, J., Jimenez, C., Local flow topologies and scalar structures in a turbulent premixed flame, Physics of Fluids 26: 065108 (2014).

124. Chong, M., Perry, A., Cantwell, B., A general classification of three-dimensional flow fields, Physics of Fluids 2: 765-777 (1990). 
125. Martin, J., Dopazo. C., Turbulent mixing and combustion modelling, In Engineering Turbulence Modelling and Experiments 5, Edited by W. Rodi and N. Fueyo, Elsevier Science Ltd. (2002).

126. van der Heijden, F., Duin, R. P. W., de Ridder, D., Tax, D. M. J., Classification, Parameter Estimation and State Estimation: An Engineering Approach using MATLAB, John Wiley \& Sons Ltd (2004).

127. Pascucci, V., Tricoche, X., Hagen, H. Tierny, J. (Ed.), Topological Methods in Data Analysis and Visualization: Theory, Algorithms and Applications, SpringerVerlag (2011). 

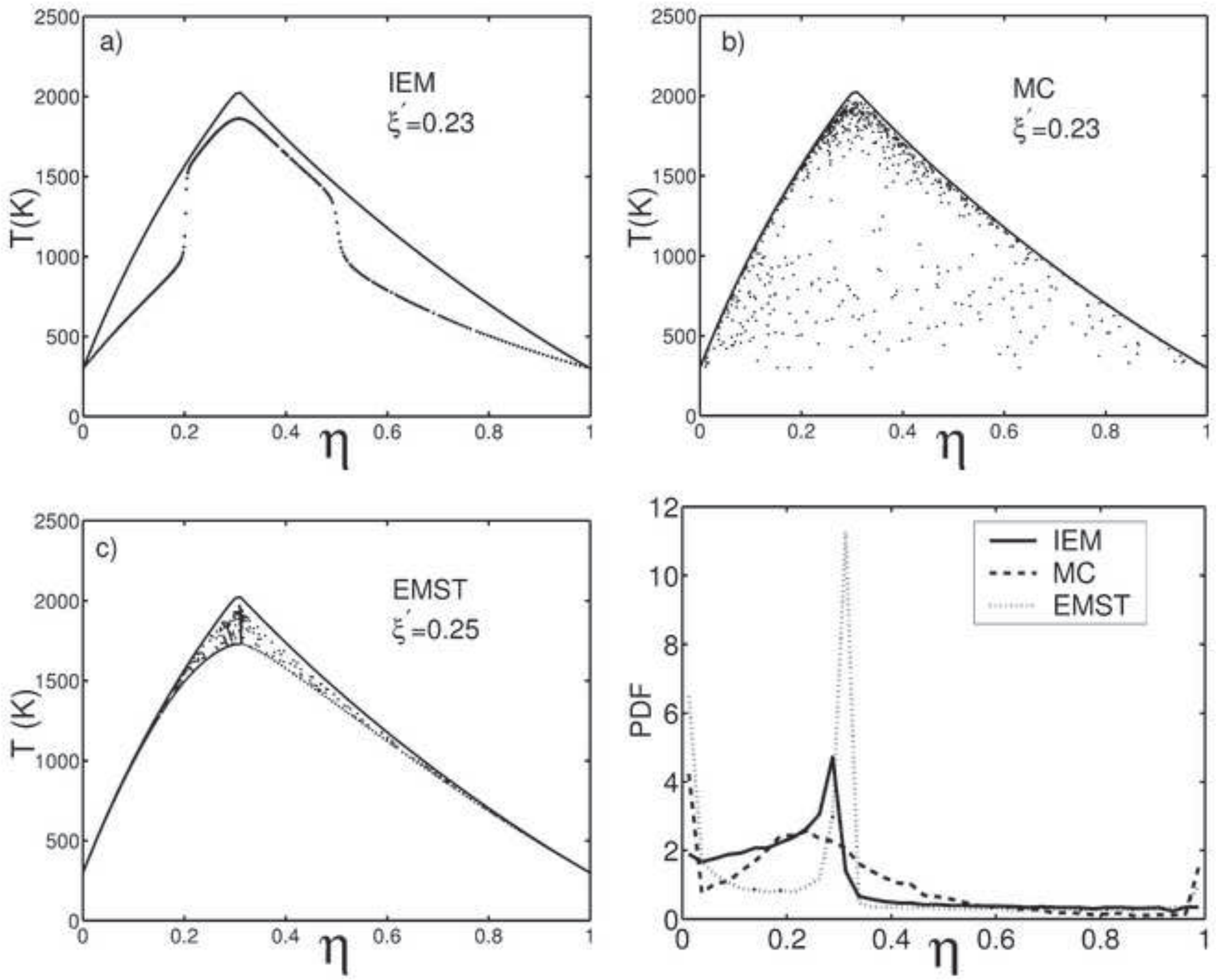


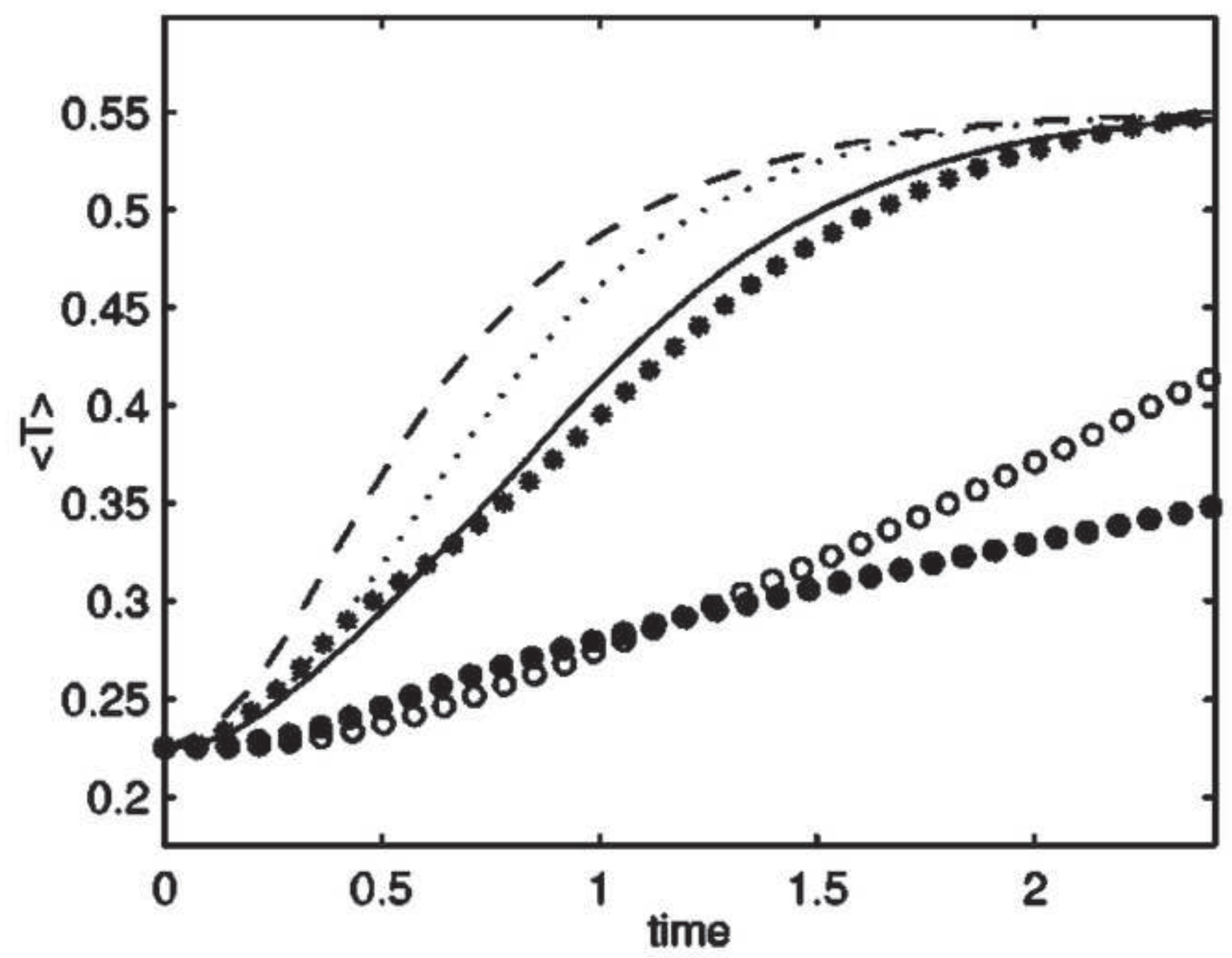




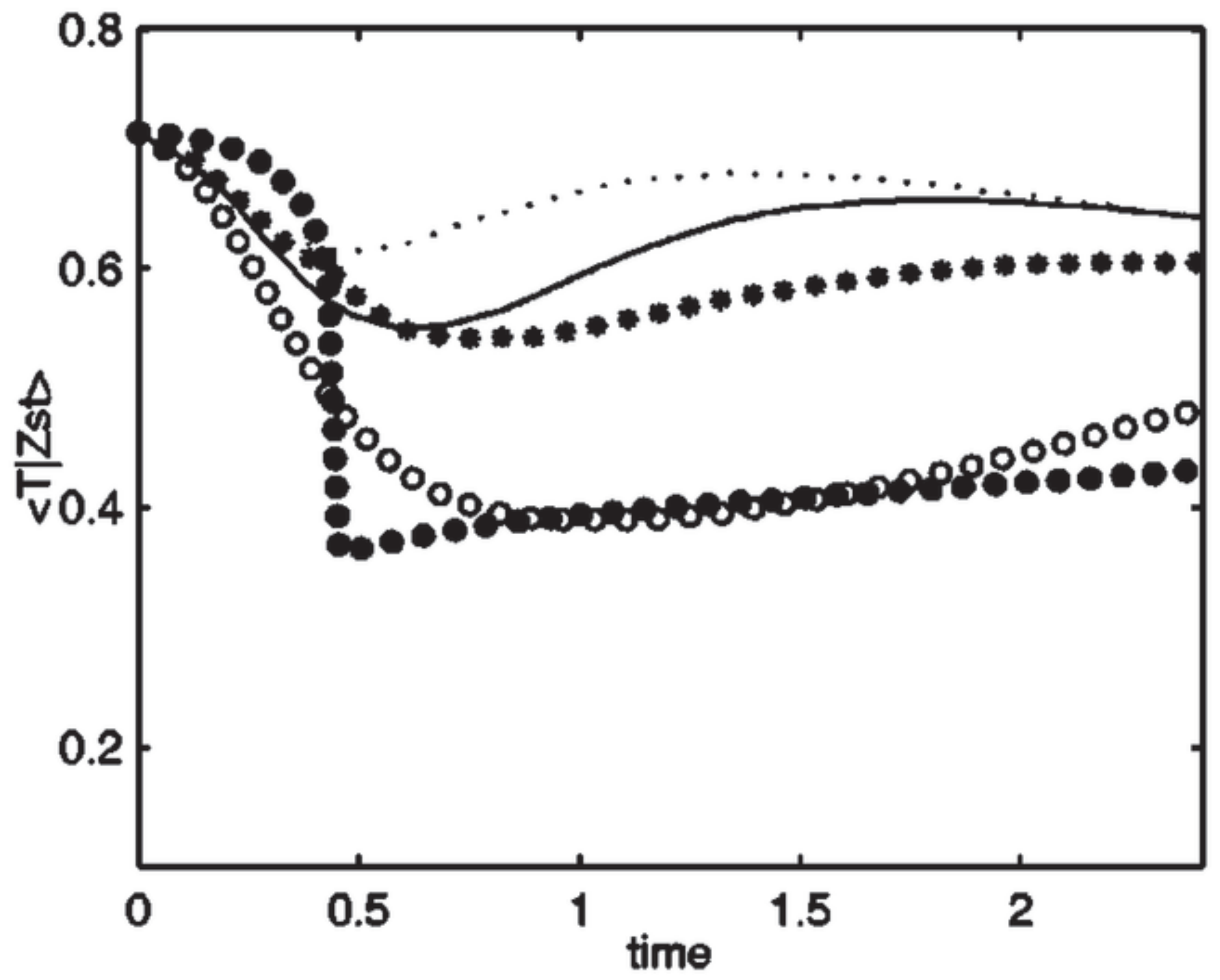



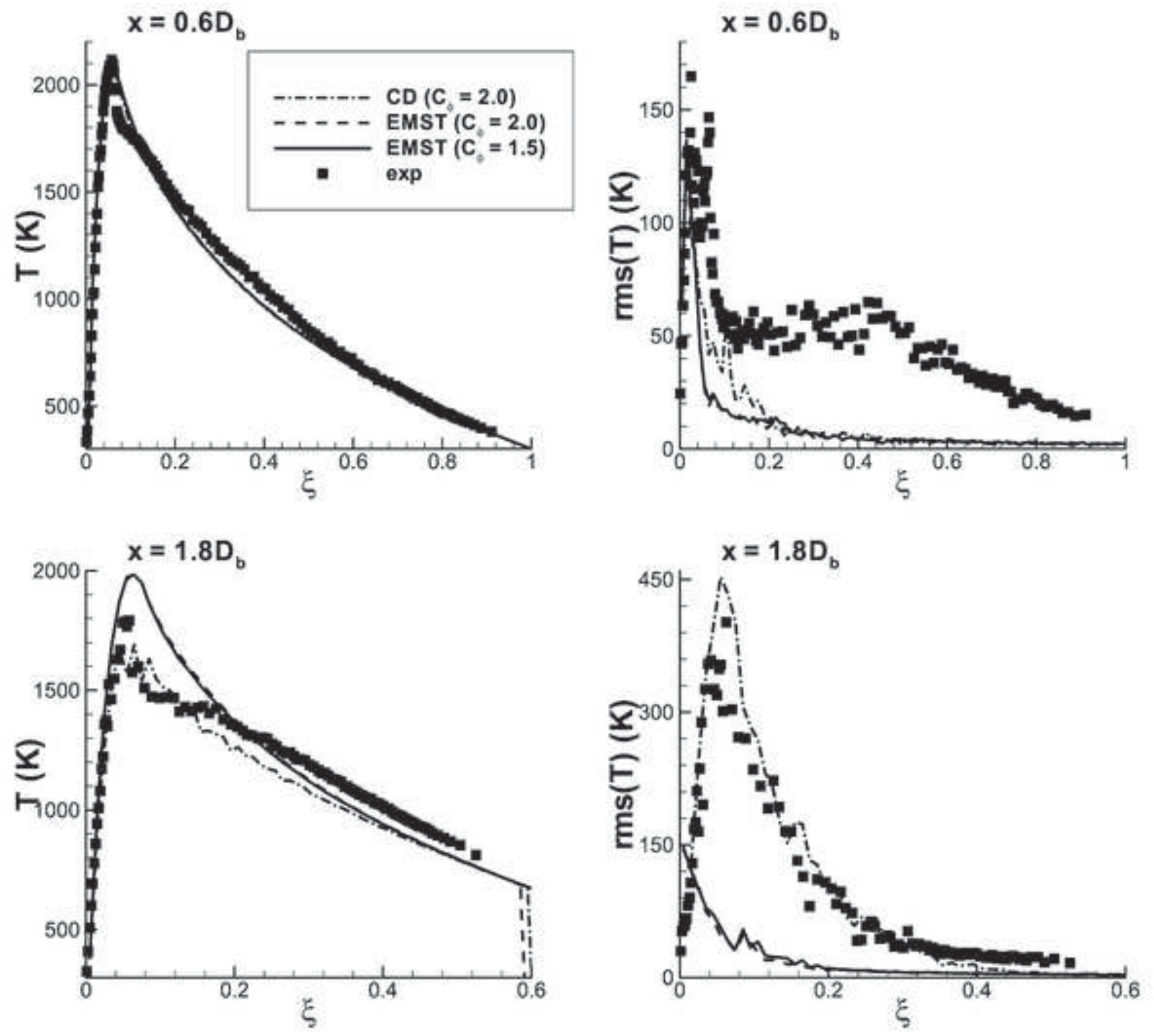
Figure4
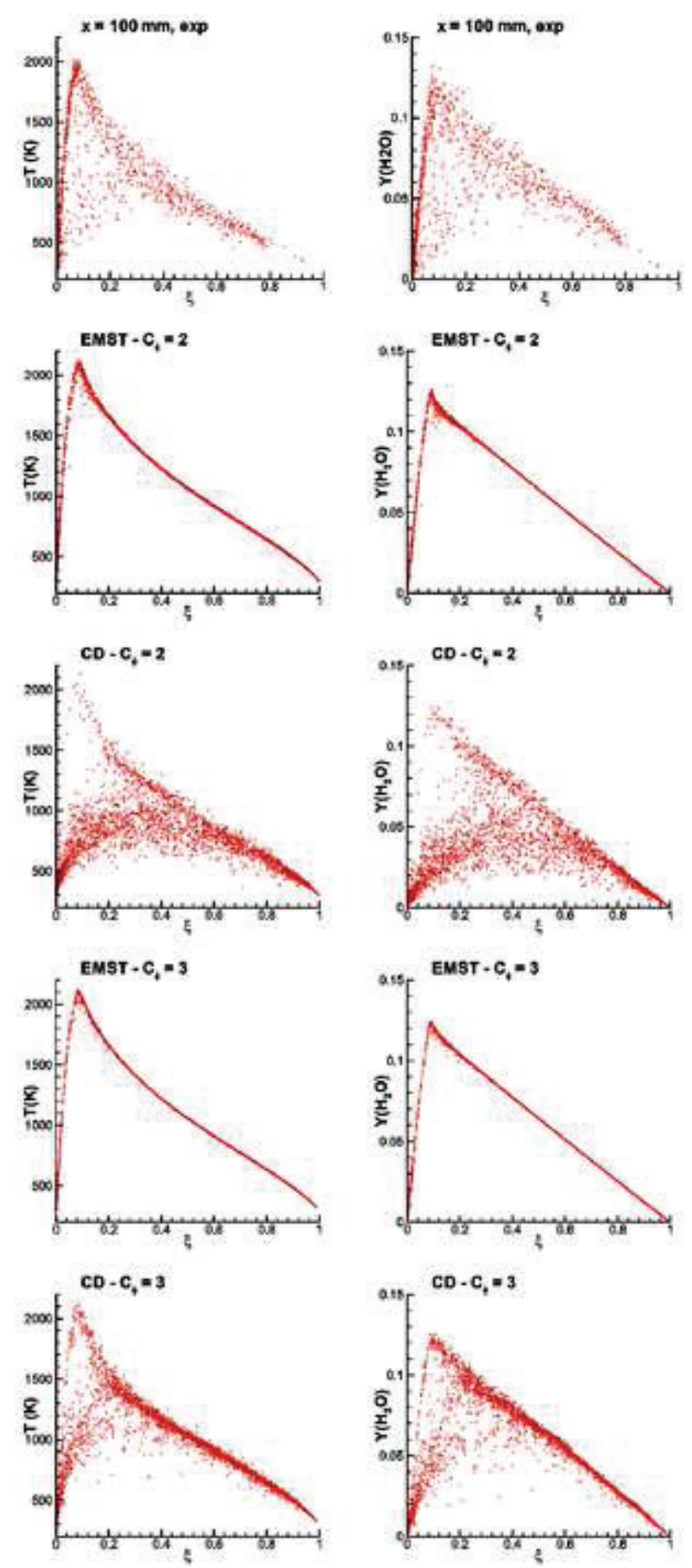


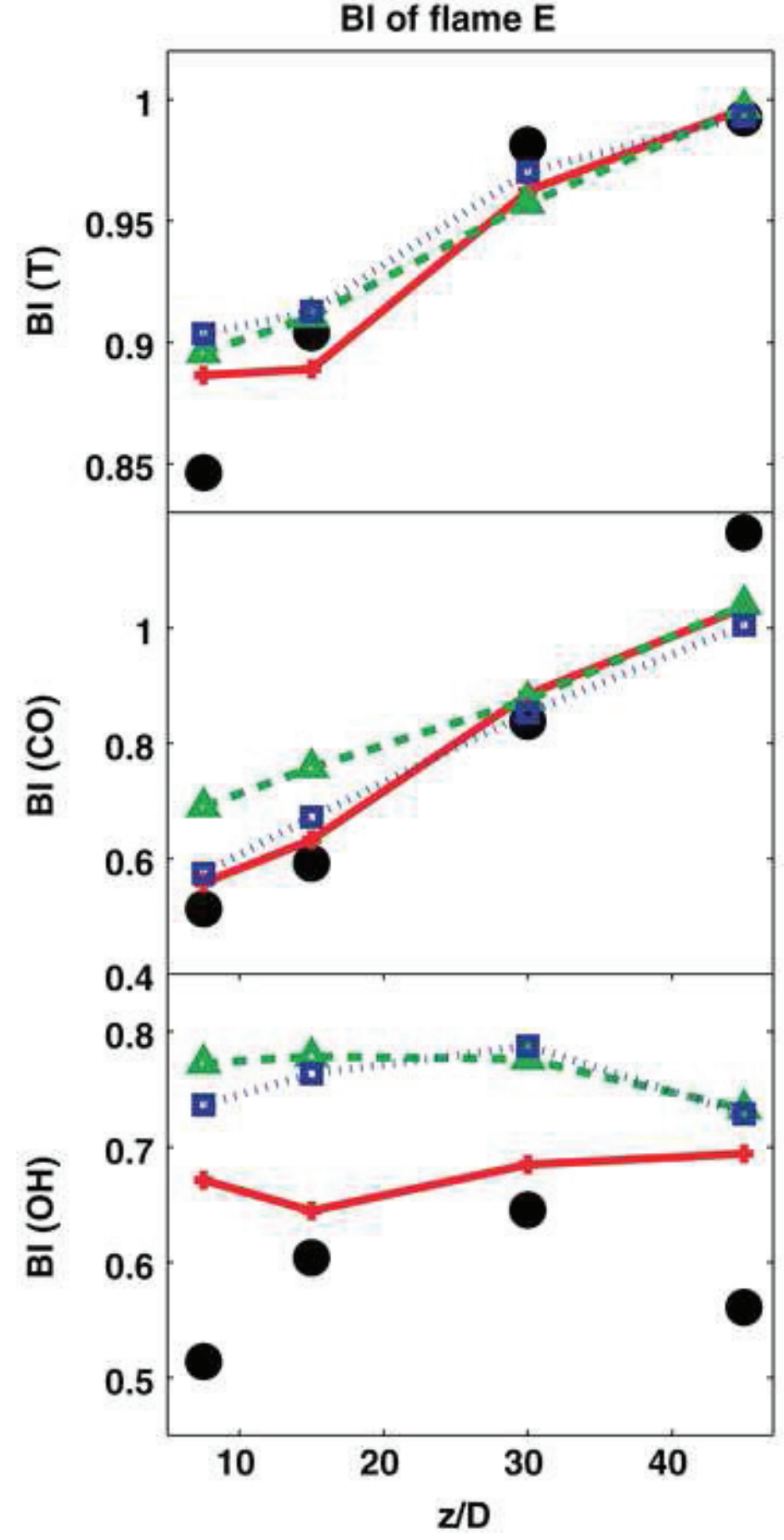



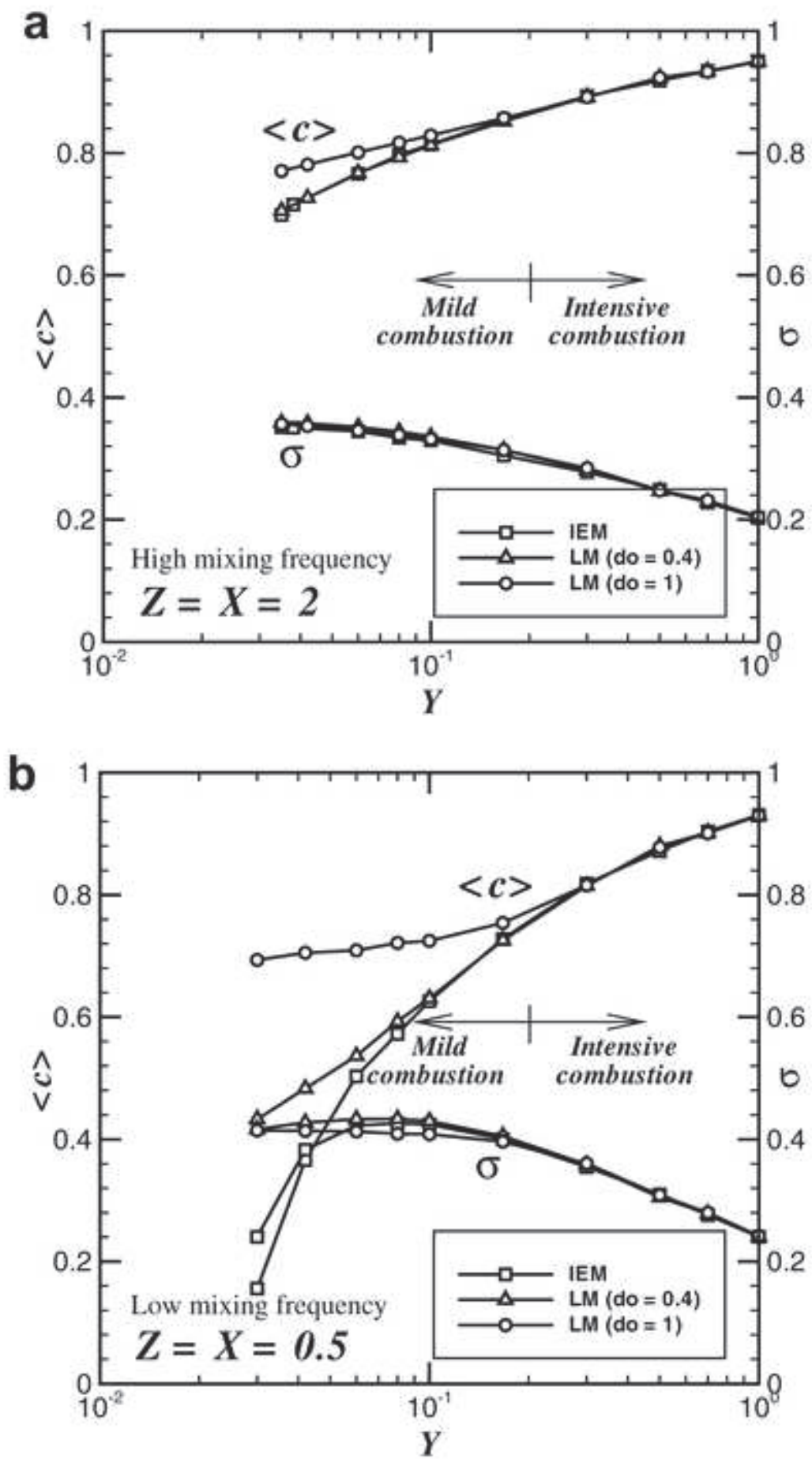


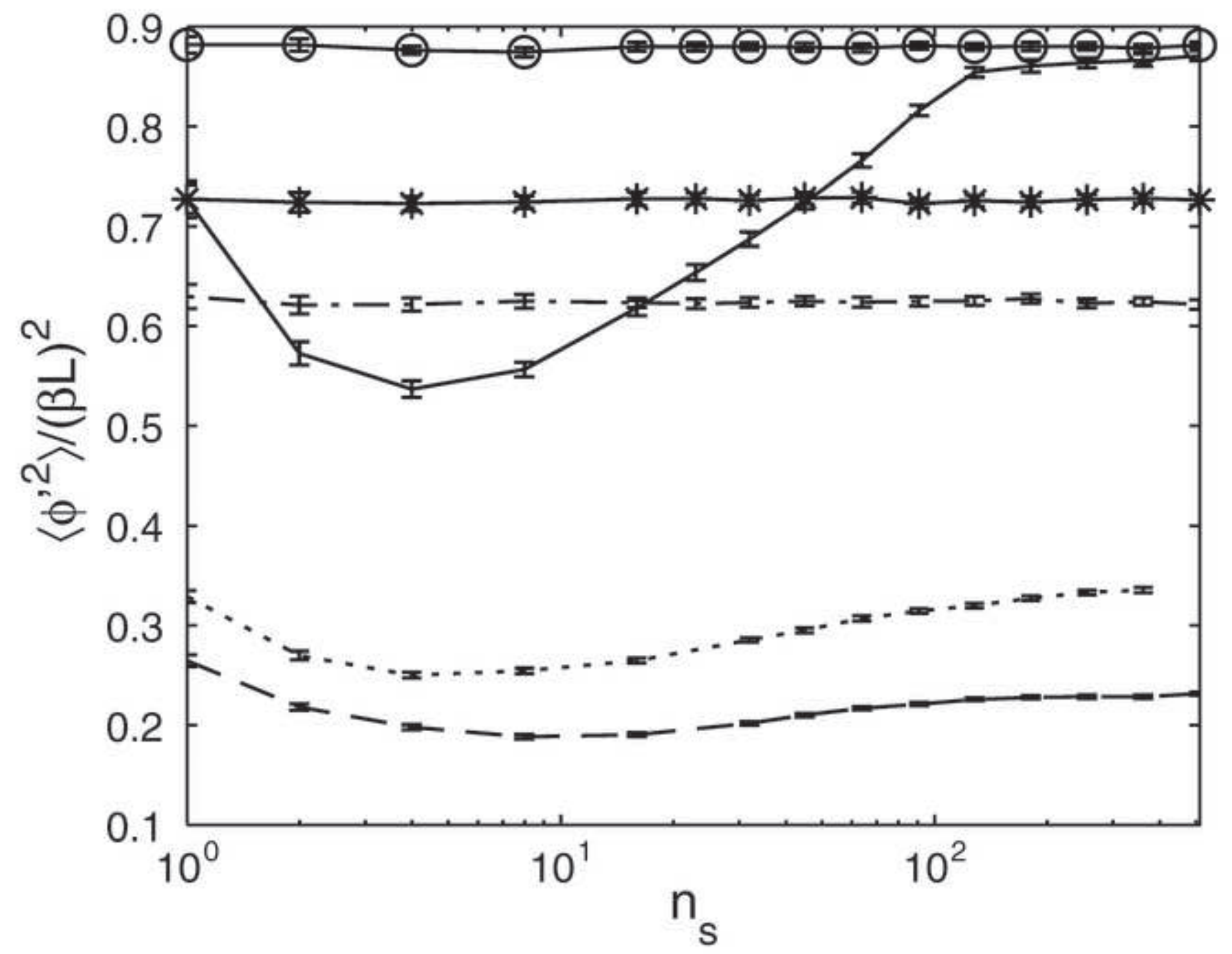




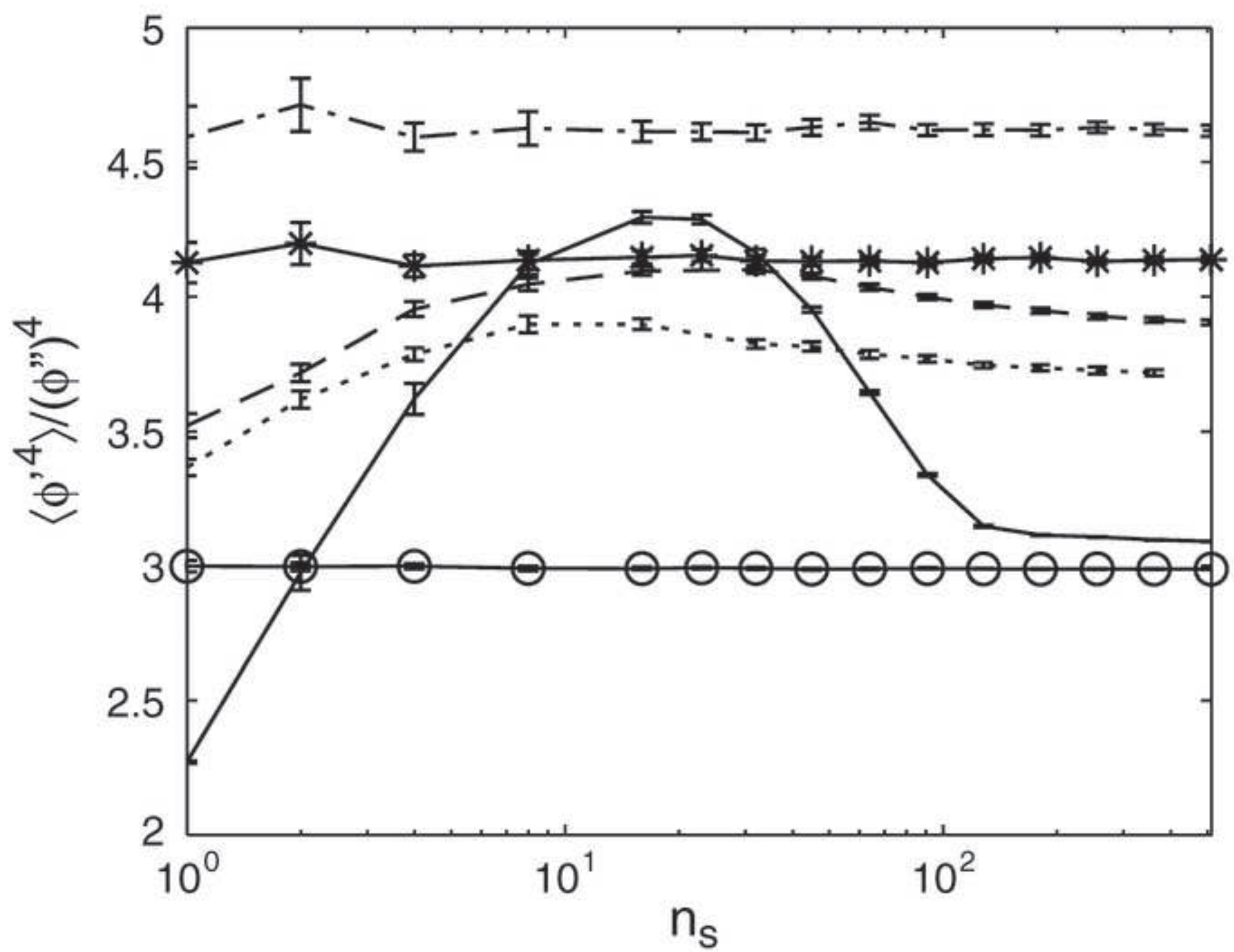




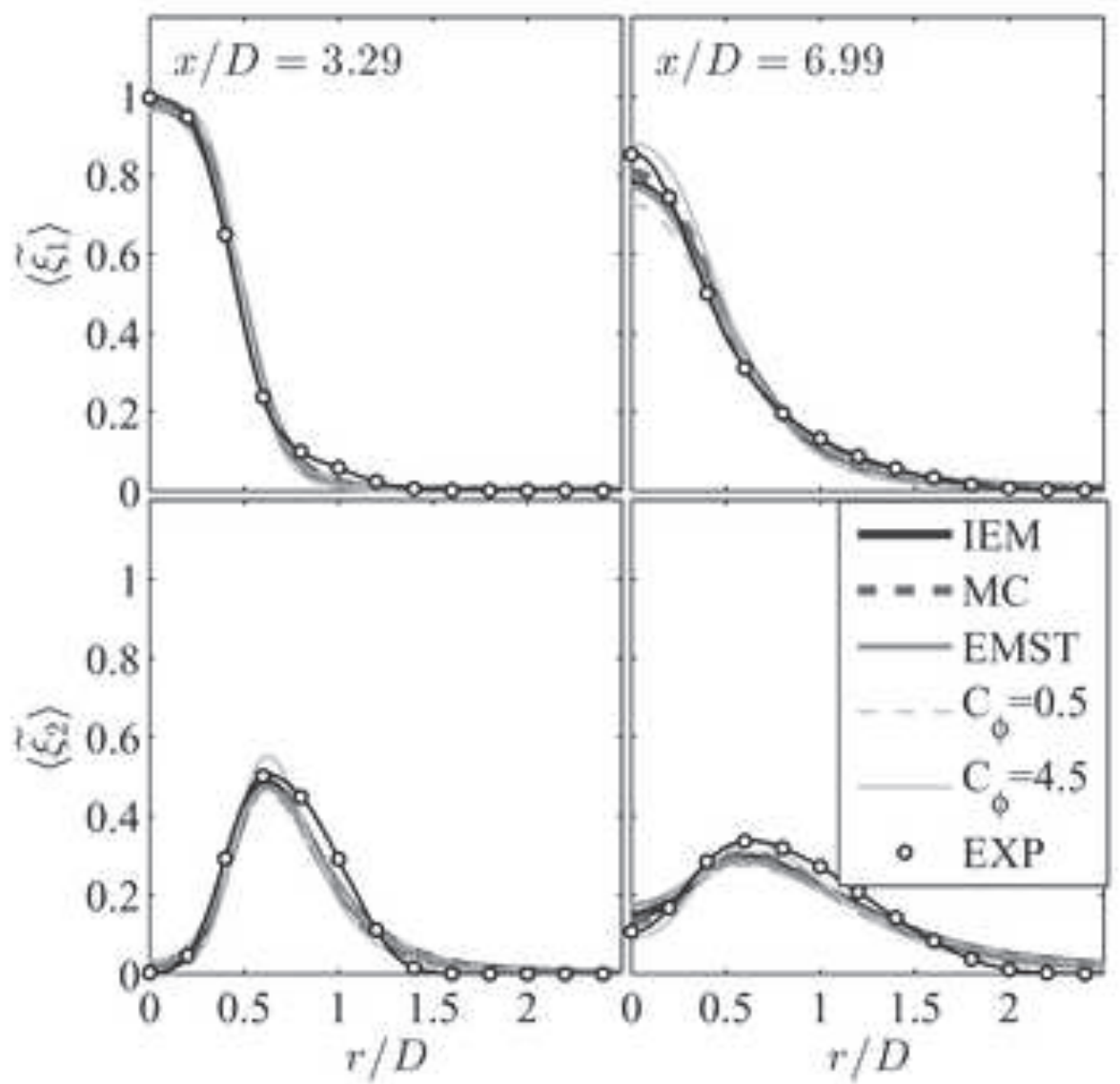

(a)

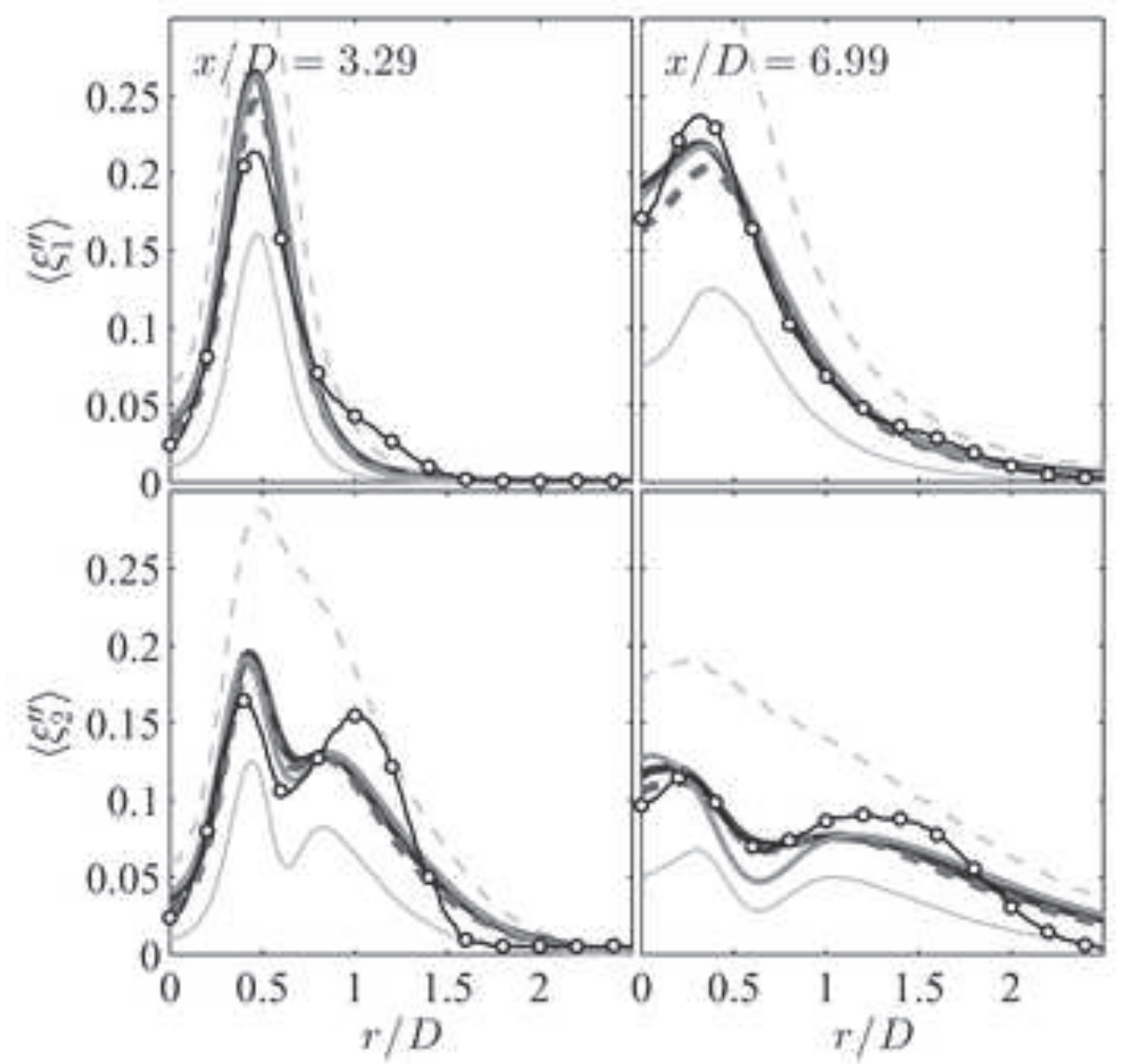

(b) 


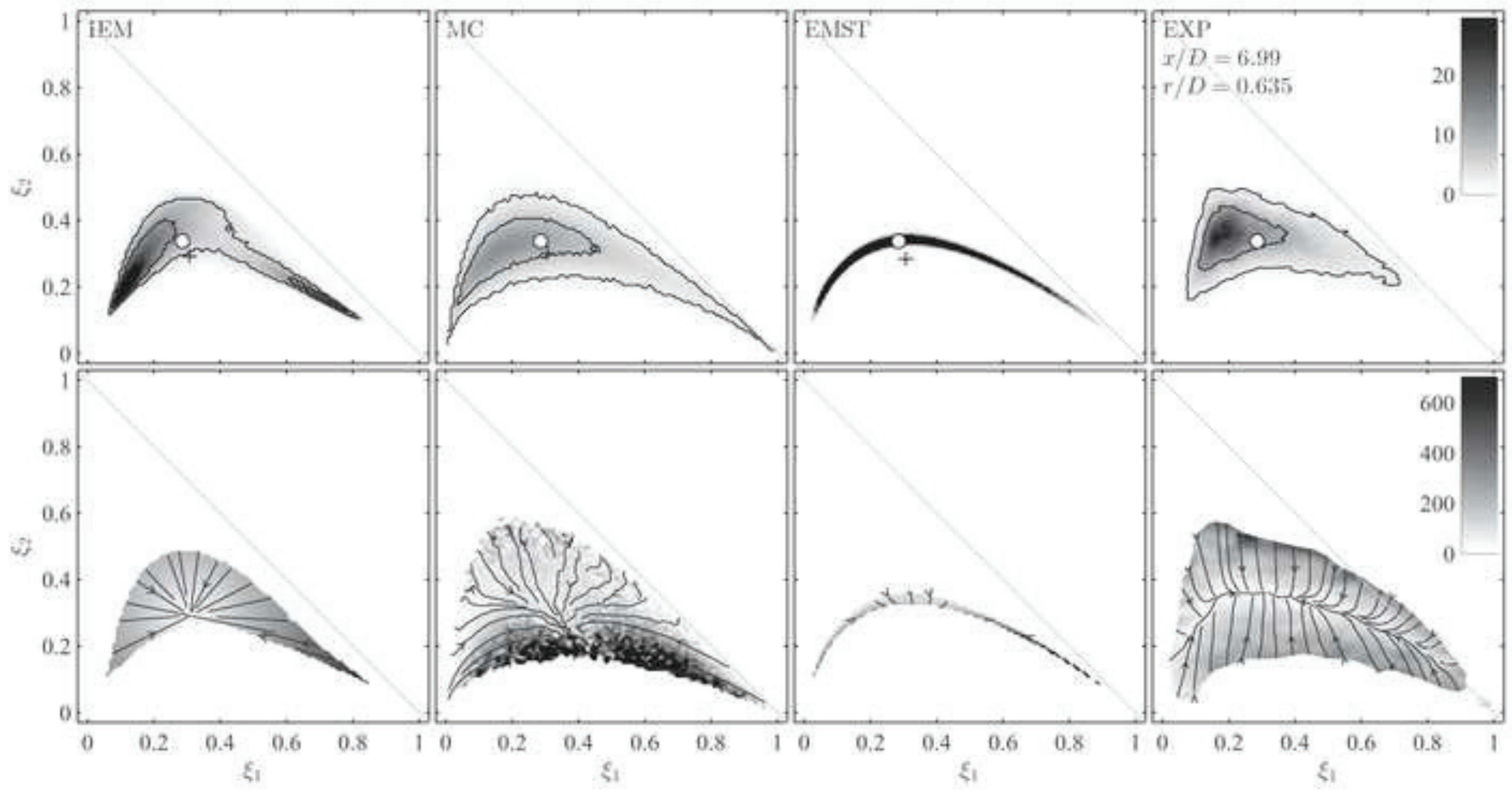

